\title{
Actuation enhances patterning in human neural tube organoids
}

\author{
Abdel Rahman Abdel Fattah (1) 1, Brian Daza (1) 1, Gregorius Rustandi', Miguel Ángel Berrocal-Rubio', \\ Benjamin Gorissen (1) 2,3, Suresh Poovathingal ${ }^{4}$, Kristofer Davie (1) ${ }^{4}$, Jorge Barrasa-Fano (1) ${ }^{5}$, Mar Cóndor (10 ${ }^{5}$, \\ Xuanye $\mathrm{CaO}^{6}$, Derek Hadar Rosenzweig ${ }^{7}$, Yunping Lei (i) ${ }^{6}$, Richard Finnell (1) ${ }^{8}$, Catherine Verfaillie (D) ${ }^{9}$, \\ Maurilio Sampaolesi (i) ${ }^{10}$, Peter Dedecker (i) ${ }^{11}$, Hans Van Oosterwyck ${ }^{5,12}$, Stein Aerts (i) ${ }^{4,13}$ \& Adrian Ranga (i) ${ }^{1 凶}$
}

Tissues achieve their complex spatial organization through an interplay between gene regulatory networks, cell-cell communication, and physical interactions mediated by mechanical forces. Current strategies to generate in-vitro tissues have largely failed to implement such active, dynamically coordinated mechanical manipulations, relying instead on extracellular matrices which respond to, rather than impose mechanical forces. Here, we develop devices that enable the actuation of organoids. We show that active mechanical forces increase growth and lead to enhanced patterning in an organoid model of the neural tube derived from single human pluripotent stem cells (hPSC). Using a combination of single-cell transcriptomics and immunohistochemistry, we demonstrate that organoid mechanoregulation due to actuation operates in a temporally restricted competence window, and that organoid response to stretch is mediated extracellularly by matrix stiffness and intracellularly by cytoskeleton contractility and planar cell polarity. Exerting active mechanical forces on organoids using the approaches developed here is widely applicable and should enable the generation of more reproducible, programmable organoid shape, identity and patterns, opening avenues for the use of these tools in regenerative medicine and disease modelling applications.

\footnotetext{
${ }^{1}$ Laboratory of Bioengineering and Morphogenesis, Biomechanics Section, Department of Mechanical Engineering, KU Leuven, Leuven, Belgium. ${ }^{2}$ J.A.Paulson School of Engineering and Applied Sciences, Harvard University, Cambridge, MA, USA. ${ }^{3}$ Department of Mechanical Engineering, KU Leuven, Leuven, Belgium. ${ }^{4}$ Center for Brain \& Disease Research, VIB-KU Leuven, Leuven, Belgium. ${ }^{5}$ Biomechanics Section, Department of Mechanical Engineering, KU Leuven, Leuven, Belgium. ${ }^{6}$ Center for Precision Environmental Health, Department of Molecular and Cellular Biology, Baylor College of Medicine, Houston, TX, USA. ${ }^{7}$ Division of Experimental Surgery, McGill University, Montreal, Canada. ${ }^{8}$ Center for Precision Environmental Health, Departments of Molecular and Human Genetics, Molecular and Cellular Biology and Medicine, Baylor College of Medicine, Houston, TX, USA. ${ }^{9}$ Stem Cell and Developmental Biology, Departments of Development and Regeneration, KU Leuven, Leuven, Belgium. ${ }^{10}$ Laboratory of Translational Cardiomyology, Department of Development and Regeneration, Stem Cell Institute, KU Leuven, Leuven, Belgium. ${ }^{11}$ Laboratory for Nanobiology, Biochemistry, Molecular and Structural Biology Division, Department of Chemistry, KU Leuven, Leuven, Belgium. ${ }^{12}$ Prometheus Division of Skeletal Tissue Engineering, KU Leuven, Leuven, Belgium. ${ }^{13}$ Laboratory of Computational Biology, Department of Human Genetics and VIB-KU Leuven Center for Brain \& Disease Research, Leuven, Belgium.

凶email: adrian.ranga@kuleuven.be
} 
G enerating complex and functional tissues during embryonic development relies on the action of mechanical forces that impose material deformations leading to precisely coordinated changes in tissue shape and identity ${ }^{1-5}$. These changes in shape and mechanical state serve critical and time-sensitive functional roles, for example in specifying cell fate ${ }^{6,7}$, spatially positioning stem cells ${ }^{8}$ and physically distorting the shape of morphogen fields 9 . Mechanical forces play a particularly significant role in neurulation, when an initially flat neural plate is shaped into a pseudostratified epithelial neural tube. The formation of the neural tube initiates by the furrowing of the neural plate, followed by bending at the single median hinge point and at the paired dorsolateral hinge points, and final apposition and fusion of the neural folds along the axis ${ }^{10}$. Neurulation requires both intrinsic forces generated within the neural plate by the apical constriction of cells at the midline, and extrinsic forces originating from tissues adjacent to the neural plate ${ }^{10-12}$. These extrinsic forces are driven by surface epithelium lying laterally to the neural folds and induce neural plate bending through a buckling mechanism. However, it is still unclear how neural tissues integrate mechanical cues to coordinate fate specification with patterning, growth and morphogenesis, and how mechanical cues and cell-intrinsic gene regulatory networks are linked ${ }^{13}$.

Organoids are ideal model systems to study the emergence of multicellular tissue complexity, as they recapitulate key elements of in vivo development. Current methods for building organoids usually start with structures made by cellular aggregation ${ }^{6,14}$ or bioprinting ${ }^{15-18}$ to impose a static form, and then rely on selforganization to direct cells to their correct identity and place. To design supportive $3 \mathrm{D}$ microenvironments for organoid culture, an important focus in recent years has been on engineering the stiffness, degradability and extracellular matrix (ECM) composition of synthetic extracellular matrices ${ }^{19,20}$. The functionality of such matrices has been augmented by the addition of features such as hydrolytically or light-mediated degradation profiles ${ }^{21-23}$, and platforms have been developed to explore the role of each of these features using artificial 3D extracellular arrays ${ }^{24,25}$. Although such engineered matrices have helped in supporting organoid growth, they have proven limited in guiding patterning and morphogenesis as they are unable to provide active mechanical forces in a temporally defined manner, as occurs in vivo. Indeed, attempts to generate shape-changing biological structures in vitro have largely focused on actuating cell monolayers. For example, contractile canine kidney (MDCK) cells have been engineered to pull on a collagen substrate to generate origami-like folds ${ }^{26}$ in a cell-type-specific method relying on cellmediated tractions, which cannot be temporally specified or controlled. In the context of modeling neural development, microfluidic ligand gradients have been used to generate millimeter-scale patterns in hPSC-derived cell monolayers ${ }^{27,28}$, and neuroepithelial cells have been made to adhere to mechanically activated micropatterns leading to perturbed patterns of gene expression ${ }^{29}$. These techniques have remained confined to $2 \mathrm{D}$, however, with limited flexibility in actuation mode. The importance of mechanical perturbation on $3 \mathrm{D}$ tissue has also been shown in an in vivo context, where intestinal organoids were shown to mature when transplanted into the mesentry of mice and loaded via a spring-based system ${ }^{30}$. It has nonetheless not been possible to actuate organoids in vitro, and studying how active mechanical forces regulate organoids in a controlled, developmentally biomimetic context has remained challenging.

Here, we develop a platform that can provide active mechanical forces to guide the fate, patterning and morphogenesis of organoids in 3D. We investigate the effects of matrix actuation and stiffness on floor plate (FP) patterning in human neural tube organoids (hNTOs). We further explore the transcriptomic landscape of unstretched and stretched hNTOs to reveal processes involved in mechanoregulation of the FP. Finally we perform perturbation experiments to validate our transcriptomic analysis.

\section{Results}

Stretching enhances floor plate patterning. In order to understand the role of mechanical forces on early human neural fate specification and patterning, we reasoned that a patternable 3D organoid model of neurulation would be necessary to deconstruct and manipulate the relationship between cells and forces. Morphogens such as SHH, RA, BMP4, and WNT play a determining role in specifying cellular identities in the developing neural tube $^{31}$. We have previously shown that RA alone is sufficient to pattern mouse neural tube organoids (mNTOs) derived from single mouse embryonic stem cells, but that competence to these signals is temporally restricted between the second and third day of differentiation ${ }^{25}$. To assess whether these conditions could be used to generate hPSC-derived neural tube organoids (hNTOs), single hPSC cells were embedded in a synthetic poly(ethylene) glycol (PEG)-based extracellular matrix whose composition and stiffness matched previously optimized properties for mNTOs. In combination with RA, we supplemented the growth medium with smoothened agonist (SAG) for FP induction, as previously shown $^{32}$. These conditions were permissive for the growth of multicellular pseudostratified epithelial organoids (Fig. 1a and Supplementary Fig. 1a). We assessed neural patterning in these organoids at day 11 by immunohistochemistry for FOXA2, a marker of the ventral-most FP domain, and found that the optimal competence window for FP induction and patterning for hNTOs occurred later than in mNTOs, between day 3 and day 5 , consistent with reports of delayed differentiation between mouse and human in vitro development ${ }^{33}$ (Fig. 1a and Supplementary Fig. 1b). Patterning was assessed using a combination of FOXA2 area ratio (AR) and FOXA2 intensity (see methods).

To apply extrinsic mechanical forces on these organoids, we designed a process that enabled the programmable actuation of gel-embedded hNTOs. Taking advantage of the adhesion between PEG-based hydrogels and silanized silicone, we transferred strains from a stretchable silicone membrane to growing organoids within crosslinked PEG using an instrument equipped with programmable equibiaxially actuated arms (Fig. 1b). As mechanical forces during the initiation of neural tube bending and closure occur simultaneously with patterning induction via notochord-derived signaling ${ }^{34}$, we reasoned that the initiation of organoid actuation could occur at the same time as morphogen treatment. The actuation of organoids was therefore initiated at day 3 , gradually increased to $100 \%$ area $(41 \%$ radial strain) by day 5 and was thereafter maintained until the experiment endpoint at day 11. Actuation resulted in a $30 \%$ increase in organoid size compared to unstretched controls (Fig. 1c). The number of cells per organoid was proportional to organoid size, demonstrating that the observed increase in size was due to higher proliferation rather than to a decrease in cell density (Supplementary Fig. 2ab). This observation was confirmed by EdU staining, with more proliferative cells seen in stretched hNTOs, located mainly at the basal side of the organoid (Supplementary Fig. 2c).

In order to understand the role of stretching on organoid differentiation and patterning, the number of organoids containing cells with FOXA2+ FP identity, as well as those exhibiting a patterned FOXA2+ domain was quantified. The proportion of organoids in which FOXA2+ cells could be detected was $~ 60 \%$ in both conditions, indicating that stretching did not alter the incidence of cells with FP identity. Strikingly, actuation enhanced the proportion of organoids with a patterned FOXA2+ domain, 


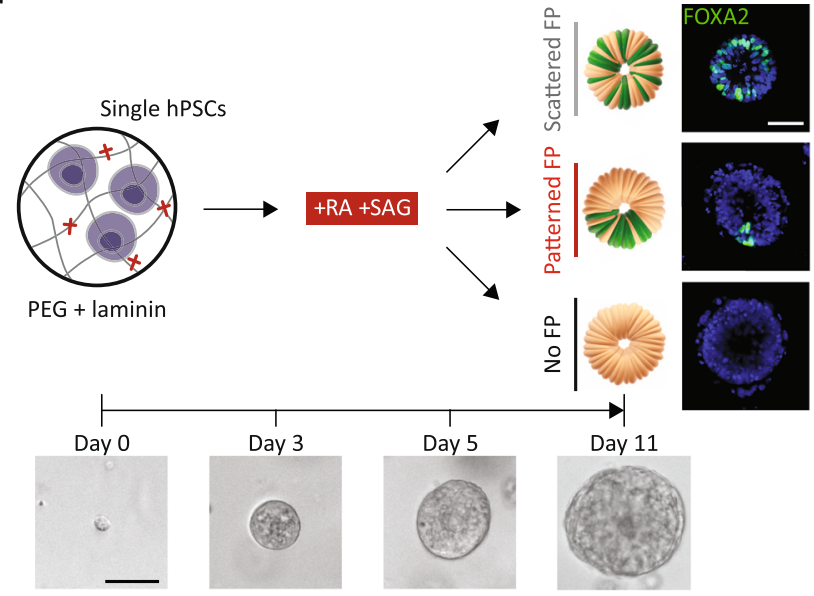

c
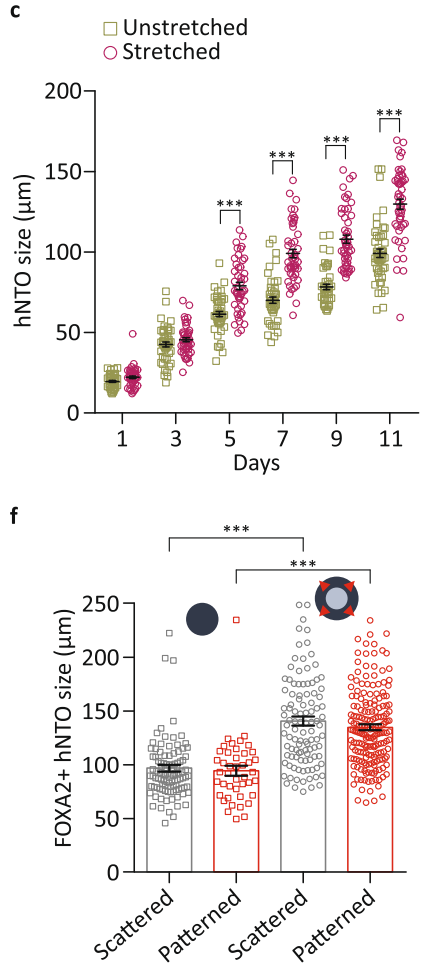
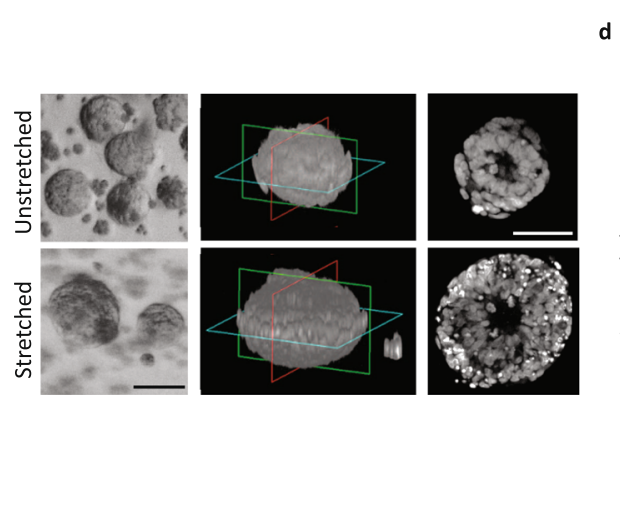

g

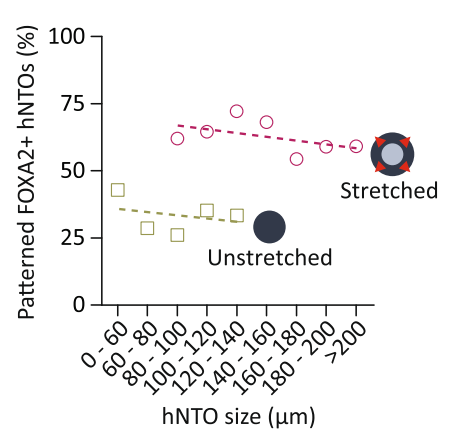

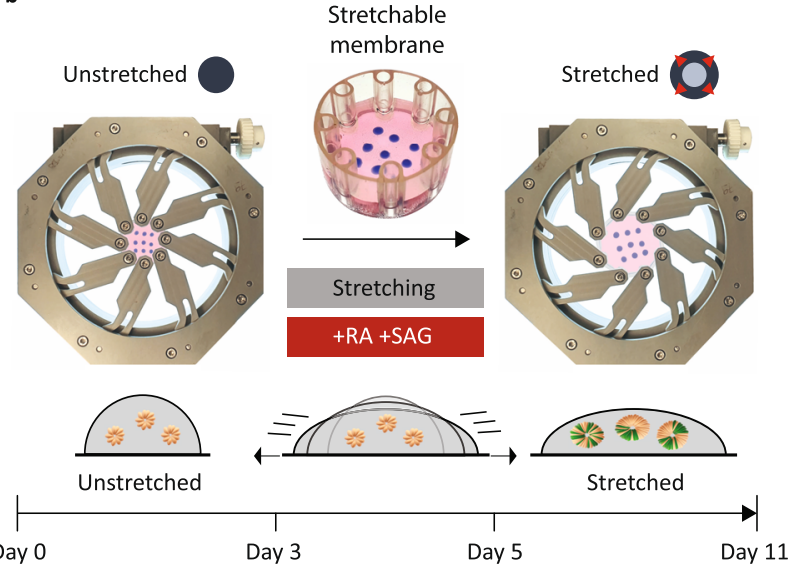
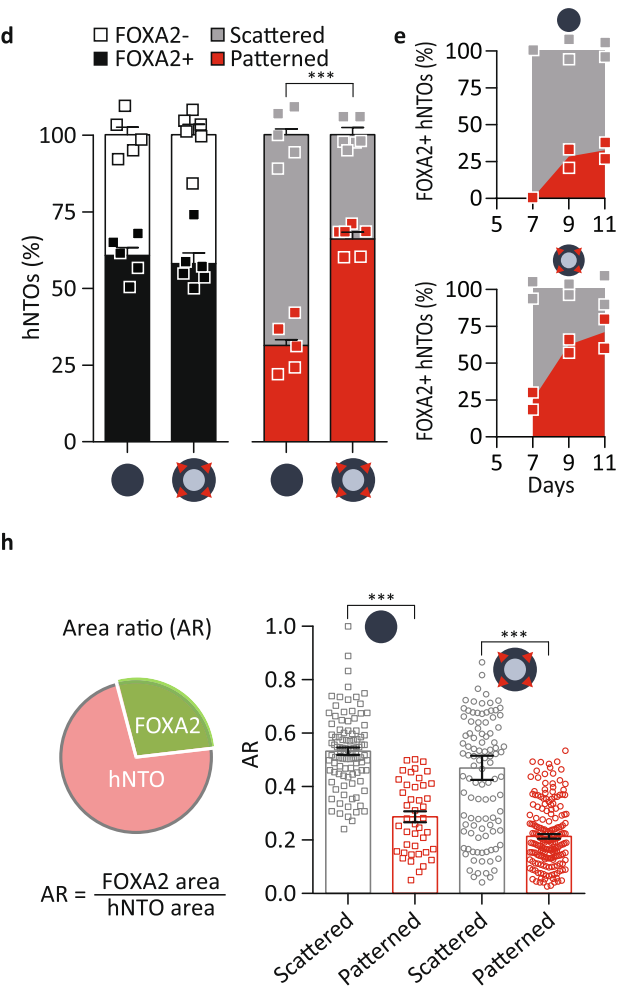

Fig. 1 Organoid growth and patterning modulation through matrix actuation. a Schematic representation of the hNTO differentiation protocol. Floor plate induction by RA-SAG treatment, with organoid phenotype categorized based on scattered, patterned or absence of FOXA2 expression. Representative images of hNTOs at days $0,3,5$, and $11(n=3)$. b Actuation of hNTOs. Hydrogel droplets (shown in blue for visualization) in stretchable membrane attached to the arms of an equibiaxial actuation device. Stretching is imposed simultaneously with RA-SAG treatment (days 3-5), with membrane surface area reaching 100\% area increase at day 5 and maintained thereafter. c Increase in hNTO size by stretching at day 5 until day 11 ( $n=3$ for a total of 50 hNTOs per datapoint, statistical analysis was determined by two-way ANOVA with Fisher's LSD test for multiple comparisons (day $5 p<0.0001$, day 7 $p<0.0001$, day $9 p<0.0001$, day $11 p<0.0001)$. Representative brightfield images, 3D confocal reconstructions and corresponding optical sections $(n=$ 3). d Quantification of FP induction and pattering events in FOXA2+hNTOs ( $n=5$ for unstretched ( 303 hNTOs) and $n=6$ for stretched ( 480 hNTOs) conditions, statistical analysis was determined by unpaired two-sided $t$-test $(p<0.0001)$ ). e Quantification of FOXA2 dynamics from day 7 ( $n=2$ for a total of $>20$ hNTOs per day). $\mathbf{f}$ Quantification of organoid size for stretched and unstretched conditions, binned for scattered and patterned hNTOs (hNTOs from d, statistical analysis was determined by unpaired two-sided $t$-test with Welch's correction (scattered $p<0.0001$, patterned $p<0.0001$ )). $\mathbf{g}$ Patterned hNTO frequency as a function of binned organoid size for stretched and unstretched conditions (hNTOs from d). $\mathbf{h}$ Area ratio (AR) quantifying the ratio between FOXA2+ domain area and total organoid area, for scattered and patterned hNTOs within each condition (hNTOs from d, statistical analysis was determined by unpaired two-sided $t$-test with Welch's correction (unstretched $p<0.0001$, stretched $p<0.0001$ )). Error bars are SEM, scalebars $50 \mu$ m.

from $25 \%$ in the unstretched control ( $303 \mathrm{hNTOs)}$ to $60 \%$ in the stretched condition (480 hNTOs) (Fig. 1d and Supplementary Fig. 3a). FOXA2 expression was more abundant in unstretched condition representing an average area ratio $\mathrm{AR}_{\text {avg }}=0.46$ compared to $\mathrm{AR}_{\mathrm{avg}}=0.29$ upon stretch (Supplementary Fig. $3 \mathrm{~b}$ ).
In both conditions, FOXA2 expression was first observed 2 days after the end of the RA-SAG treatment. FOXA2 expression was initially scattered, but subsequently FP patterning increased, particularly for stretched hNTOs (Fig. 1e). We additionally confirmed that it was not the culture of the organoids within the 
device but rather stretching which led to enhanced growth and patterning (Supplementary Fig. 4a-c). Altogether, these results suggest that mechanical forces play an important role in specifying spatial relationship between cells in early hNTO development.

In order to determine whether stretching biased hNTOs via a size-dependent mechanism, we quantified scattered versus patterned organoid sizes in each condition. Whereas organoids were on average larger in stretched conditions, there were no significant size differences within each condition between organoids with scattered or patterned FOXA2 expression (Fig. 1f). Moreover, the increased frequency of FP patterning upon stretch was maintained across hNTO sizes (Fig. 1g), indicating that, within the tested conditions, organoid size does not determine patterning frequency.

The patterning of the domains within the neural tube has been reported to scale with growing tissue size ${ }^{35}$ and between species ${ }^{36}$. In order to explore whether these observations could be recapitulated with hNTOs we quantified the proportion of the projected organoid area occupied by the FP domain (Fig. 1h). For patterned organoids, the FOXA2+ domain represented $\sim 20 \%$ of the total area, whereas in unpatterned organoids, FOXA2 expression covered $50 \%$ of the total. These observations were maintained across size and stretch conditions, suggesting that FP patterning in hNTOs is a scalable process as in vivo, and that organoid stretching enhances patterning frequency without disrupting pattern scalability.

Actuation modulation and matrix stiffness tune floor plate patterning. To gain insights into the role of force magnitudes and duration on hNTO patterning, we modulated the extent and time of hNTO exposure to stretch. FP patterning efficiency increased with stretch magnitude and decreased when initiation of stretch was delayed, or by early removal from the stretching device (Fig. 2a). In particular, we observed a linear dependency on stretch magnitudes and a mechanical temporal competence window between days 3 and 7 when mechanical stimulation had the greatest and irreversible effect on enhancing FP patterning. These observations suggest that hNTOs retain a mechanical memory of applied forces, in line with emerging experimental ${ }^{37}$ and theoretical ${ }^{38}$ results in other systems. To test whether stretch direction would bias the direction or frequency of patterning, we built a device, which allowed us to stretch organoids uniaxially at the same rate as that applied with the equibiaxial device. Increased FP patterning was observed in uniaxially stretched hNTOs, similarly to organoids stretched equibiaxially; however, no preferential patterning orientation was observed, indicating that force magnitude rather than directionality is involved in the enhanced hNTO patterning phenomena (Supplementary Fig. 5a-f).

We and others have previously shown that the stiffness of the local microenvironment can modulate organoid patterning and morphogenesis ${ }^{23,25}$. To deconvolve the role of active (actuated) versus passive (matrix) mechanics on FP patterning, we stretched hNTOs embedded in soft $(0.7 \mathrm{kPa})$, intermediate $(2 \mathrm{kPa})$ and stiff $(8 \mathrm{kPa})$ PEG hydrogel matrices. FP patterning efficiency was only enhanced at intermediate matrix stiffness and remained unchanged upon stretching in soft and stiff matrices (Fig. 2b). In parallel, we observed that while organoids increase in size at the fastest rate in the softest matrix, a relative increase in size upon stretch was only evidenced at intermediate $2 \mathrm{kPa}$ stiffness (Fig. 2c). These results suggest a dynamic mechanical feedback whereby organoid growth compresses the adjacent matrix, with the concomitant matrix resistance counteracting organoid growth and that the same feedback is likely involved in pattering enhancement upon stretch.

To interrogate the effects of such changes to the mechanical microenvironment on organoid growth in actuated conditions, we assessed matrix stretch in the direction of stretch $\left(\lambda_{\mathrm{rr}}\right)$. A stress measure $\left(\sigma_{\mathrm{rr}}\right)$ was then calculated using a Neo-Hookean incompressible material model (Supplementary Fig. 6a, Fig. 3a, see methods). In unstretched conditions, we observed matrix compression $\left(\lambda_{\mathrm{rr}}<1, \sigma_{\mathrm{rr}}<0\right)$ driven by hNTO growth (Fig. 3a). In matrix regions devoid of hNTOs, stretching generated tensile strains and stresses $\left(\lambda_{\mathrm{rr}}>1, \sigma_{\mathrm{rr}}>0\right)$. Finite element analysis simulations of equibiaxial stretching produced similar stresses, $\sigma_{\mathrm{rr}, \text { simulation }}$, as those assessed experimentally, $\sigma_{\mathrm{rr}, \text { experiment }}$ (Supplementary Fig. 6b). The simulations further confirmed that the generated tension field was homogeneous throughout most of the matrix volume where organoids are evaluated (closest to the bottom of the gel droplet), with the exception of the outermost edge regions of the hydrogel, which in experiments are largely devoid of hNTOs (Supplementary Fig. 6c). In matrix regions containing hNTOs, organoid growth-induced compression was greater than stretch-induced tension, leading to the conclusion that actuation did not impose net tension stresses on hNTOs (Fig. 3a). Instead, stretching provided relief of growth-induced compression in the vicinity of the hNTOs, such that $\lambda_{\text {rr,stretched }}>$ $\lambda_{\text {rr,unstretched }}$ and $\sigma_{\text {rr,stretched }}>\sigma_{\text {rr, unstretched }}$ for days $\geq 5$ (Fig. 3a). To investigate whether the magnitude of compressive stresses drive organoid growth, we analyzed the relationship between $\sigma_{\mathrm{rr}, \text { Day11 }}$ and hNTO radius growth rates. A fast $(>4 \mu \mathrm{m} /$ day $)$ and a slow $(<4 \mu \mathrm{m} /$ day $)$ growth regime were observed, which were separated by a critical stress $\sigma_{\text {rr,critical }}=-6.19 \mathrm{kPa}$ (Fig. 3b).

Because soft matrices exert little counteracting pressure on organoid growth, $\sigma_{\mathrm{rr}, \text { Day11 }}>\sigma_{\mathrm{rr}, \text { critical }}$ in both stretched and unstretched conditions (Fig. 3c) and hNTOs therefore have similarly high growth rates and large sizes (Fig. 2c). Conversely, in stiff matrices, although actuation relieves significant stress, both conditions result in $\sigma_{\mathrm{rr}, \text { Day11 }}<\sigma_{\mathrm{rr} \text {,critical, and thus hNTOs }}$ have similar low growth rates resulting in small hNTOs. In contrast to soft and stiff matrices, unstretched intermediate matrices have $\sigma_{\mathrm{rr} \text {,Day11 }}<\sigma_{\mathrm{rr} \text {,critical }}$, corresponding to slow growth conditions. Upon stretch, a transition to the faster growth regime occurs where $\sigma_{\mathrm{rr} \text {,Day11 }}>\sigma_{\text {rr,critical }}$, resulting in faster growth rates (Fig. 2c). These growth rate variations are linked to cell proliferation: by staining for EdU we found high cell proliferation in hNTOs embedded in $0.7 \mathrm{kPa}$ hydrogels under stretched and unstretched conditions as well as those in stretched $2 \mathrm{kPa}$ hydrogels (Supplementary Fig. 7). While these conditions share a high growth rate regime with $\sigma_{\mathrm{rr}, \text { Day11 }}>\sigma_{\mathrm{rr}, \text { critical }}$, it is only in the latter condition that FOXA2 patterning occurs at high frequency. Therefore, a high growth rate regime does not by itself impose higher patterning frequency. Rather, a transition from low to high growth rate regimes, only possible in matrices of intermediate stiffness, appears necessary for enhancing FP patterning. This suggest that a matrix stiffness matching that of the in vivo microenvironment is not only permissive to growth and cellular reorganization, but also functions as a substrate for optimal transfer, at high fidelity, of extrinsic forces that lead to FP patterning enhancement. Moreover, the growth rate transition in actuated intermediate stiffness matrices likely alters specific cellular programs responsible for increasing FP patterning frequencies.

Transcriptional landscape of actuated hNTOs. To comprehensively define the cellular identities in hNTOs and to unravel underlying differences between control and stretched hNTOs, we performed scRNAseq on organoids at three timepoints: before 


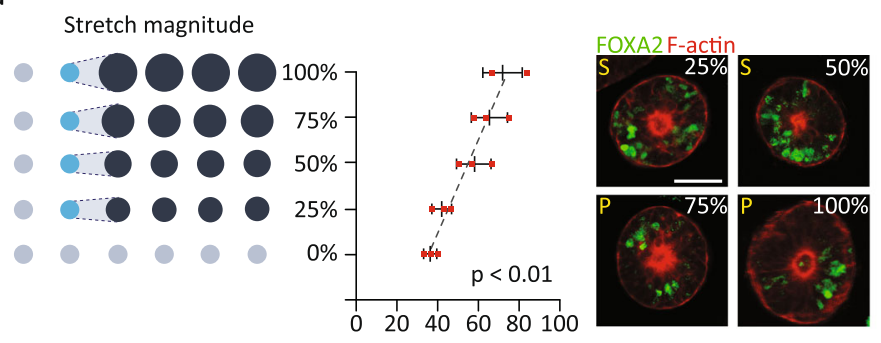

Stretch delay (SD)
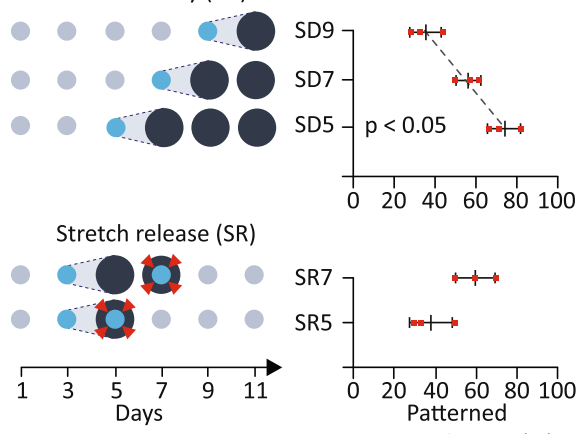
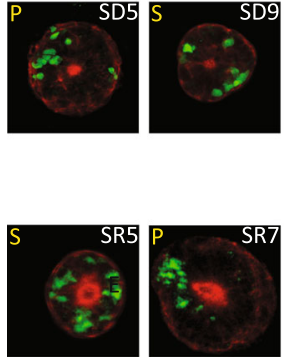

FOXA2+ hNTOs (\%)
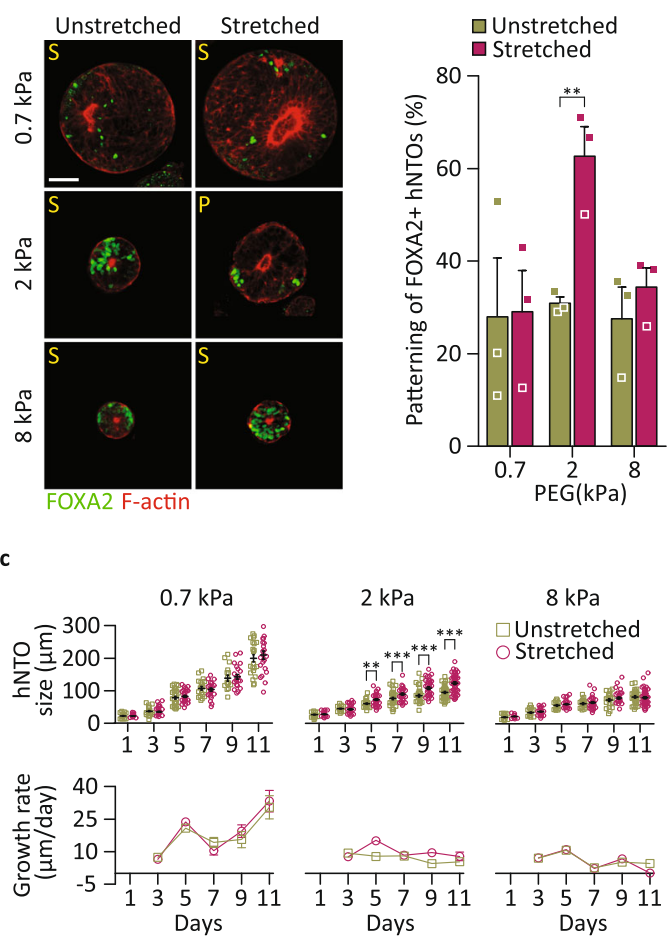

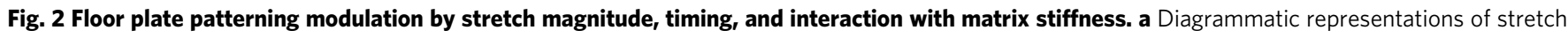
magnitude regime modulation from 0 (no stretch) to 100\% (maximal stretch, in area increase) as well as stretch delay (SD) and stretch release (SR) modulation experiments, with corresponding quantification of patterning efficiencies and representative images showing FOXA2 and F-actin expressions (Error bars are SD, center of error bars denote the mean, $n=3$ for a total of $>70$ hNTOs for all data points except for stretch magnitudes $75 \%$ ( 49 hNTOs) and 25\% (45 hNTOs), $p$-values denote Pearson correlation analysis, stretch magnitude $p=0.0018$, stretch delay $p=0.0373$ ). $\mathrm{S}$ and $\mathrm{P}$ denote scattered and patterned FP assignment, respectively. b FOXA2 and F-actin expressions in hNTOs embedded in soft ( $0.7 \mathrm{kPa}$ ), intermediate (2 kPa) and stiff ( $8 \mathrm{kPa}$ ) matrices. Quantification of stretch-induced FP patterning for each matrix stiffness condition ( $n=3$ for unstretched $0.7 \mathrm{kPa}$ ( $84 \mathrm{hNTOs}$ ), $2 \mathrm{kPa}$ ( $47 \mathrm{hNTOs)}$ $8 \mathrm{kPa}$ (164 hNTOs) and stretched $0.7 \mathrm{kPa}$ (58 hNTOs), $2 \mathrm{kPa}$ (73 hNTOs), 8 kPa (152 hNTOs), statistical analysis was determined by unpaired two-sided $t$ test $(p=0.0082)$. c Quantification of organoid sizes and growth rates in three matrix stiffnesses, with and without stretch ( $>30$ hNTOs per datapoint except for $0.7 \mathrm{kPa}(>15 \mathrm{hNTOs})$, statistical analysis was determined by two-way ANOVA with Fisher's LSD test for multiple comparisons (day 5 p = 0.0014 , day $7 p=0.0006$, day $9 p<0.0001$, day $11 p<0.0001)$ ). Error bars are SEM unless stated otherwise, scalebars $50 \mu \mathrm{m}$.

stretch (day 3), immediately after reaching final stretch (day 5), and after maturation (day 11). Graph-based clustering of the 17,826 cells retained for analysis indicated increased cluster diversity over differentiation time (Fig. 4a), which was confirmed by pseudotime analysis (Supplementary Fig. 8). We identified 11 clusters in this dataset, and annotated them to reveal groups of cells with dorsal (D-11), intermediate (I-11), ventral (V-5, V-11), forebrain (FB), neuroprogenitor (NP-5, NP-3), neural crest (NC), neural crest derivatives (NCD) identities, as well as two groups with shared identities assigned as transition clusters ( $\mathrm{T}$-a and Tb) (Fig. 4a-c, Supplementary Fig. 9a-b, Supplementary Data 1). The transcriptomic signature of human-specific neural differentiation was confirmed by multiple markers (Supplementary Fig. 10), including the presence of the earliest human neural marker $P A X 6^{39}$ observed in clusters corresponding to day 3 and day 5 , and a transition to the more differentiated neuroepithelial marker SOX1 seen by day 11 (Fig. 4d).

To track the differentiation of cells in the ventral domains, we established a geneset including FOXA2, NKX2.2, OLIG2, and NKX6.1, which was present in ventral clusters at days 5 and 11 (Fig. 4d). Single-cell regulatory network inference and clustering (SCENIC) analysis was performed on the dataset ${ }^{40}$, identifying 404 regulons representing transcription factors (TFs) and their predicted transcriptional targets containing statistically significant TF binding sites in their cis-regulatory control elements
(Supplementary Data 2). FOXA2 regulon activity was confirmed within ventrally associated clusters (Fig. 4d, Supplementary Fig. 11a-b).

In order to validate the identities determined by scRNAseq analysis and to spatially relate them to the ventral domain, we used endpoint immunohistochemistry (IHC) (Fig. 4e). hNTOs displayed $\mathrm{pV} 3 \mathrm{NKX} 2.2+$, progenitor motor neuron ( $\mathrm{pMN}$ ) OLIG2+, and ventral NKX6.1+ cells in close proximity to the $\mathrm{FP}^{41}$. Basal regions expressed ISL1/2+ MN cells and the early neuronal marker TUBB3. PAX6, also associated with intermediate domains, was observed mainly in FOXA2- organoids. Midbrain ventral marker LMX1A was co-expressed with FOXA2, while forebrain marker FOXG1 was present in FOXA2- hNTOs, in accordance with the expected lack of FP in the forebrain. Additionally, ZO1 expression confirmed the presence of apical tight junctions and colocalized with actin cables characteristic of apico-basally polarized epithelial organoids.

Overall, the transcriptional signature of stretched and control samples was similar, both at early (day 5) and late (day 11) timepoints, (Fig. 4f), suggesting that the imposed mechanical stimulation does not lead to changes in differentiation or to the emergence of additional cell fate types. In line with scRNAseq analysis, all markers visualized by IHC that were present in control conditions (Fig. 4e) were also observed in stretched hNTOs (Supplementary Fig. 12). As expected, genes involved in 


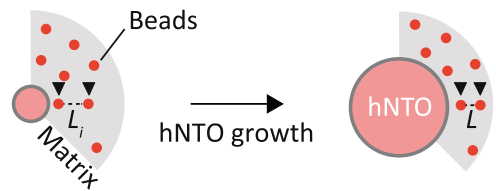

Inter-bead $\lambda_{r r}=\frac{L}{L} \quad \lambda_{r r}>1$ (Tension)

stretch $\lambda_{\mathrm{rr}}=\frac{L}{L_{i}} \quad \lambda_{\mathrm{rr}}<1$ (Compression)

$\lambda_{\mathrm{rr}}^{\mathrm{rr}}=1$ (Neutral)
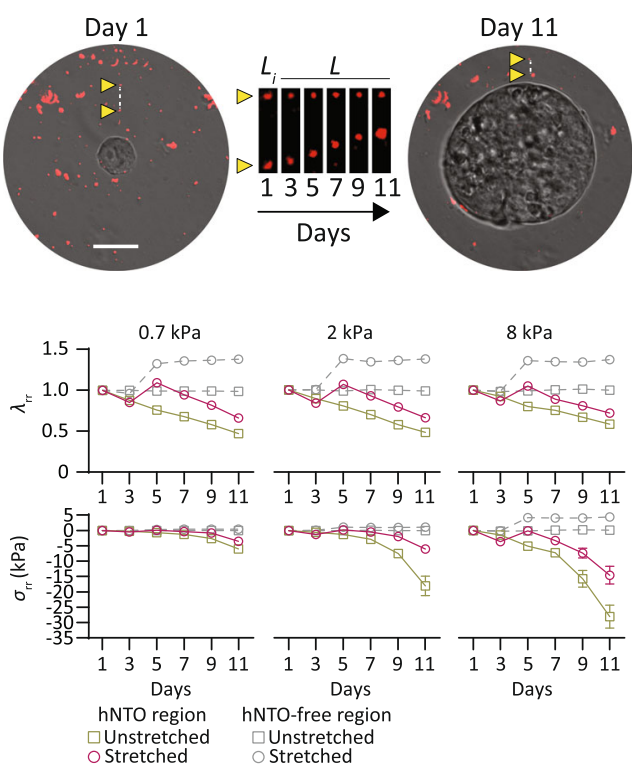
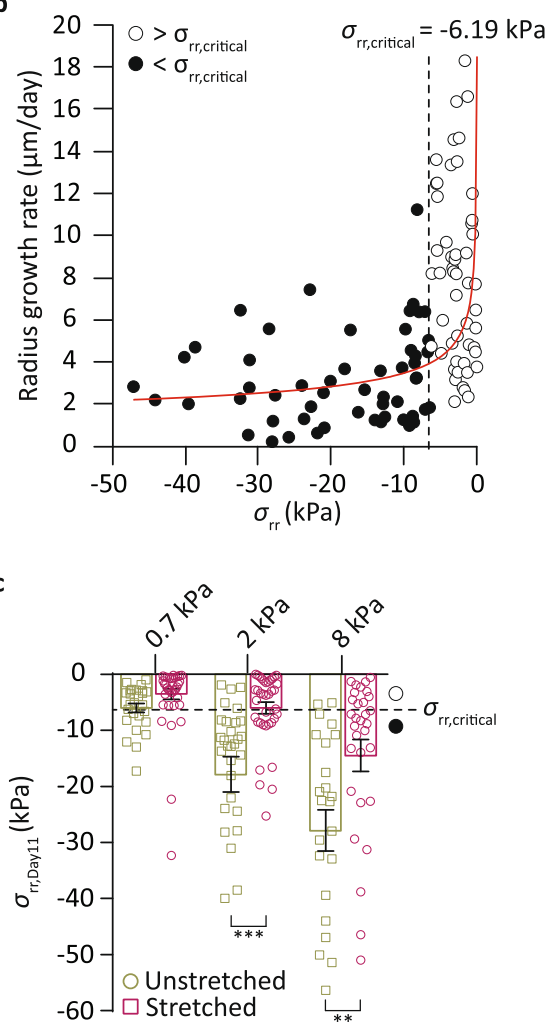

Fig. 3 Matrix actuation modulates mechanical microenvironment by relieving growth-induced compression. a Schematic representation of bead tracking technique and corresponding representative images (yellow arrows point to a representative red fluorescent bead pair) where $\lambda_{\text {rr }}$ is the interbead stretch and $L$ and $L_{i}$ denote the interbead distance and initial interbead distance, respectively. Quantification of matrix radial stretch and stress for soft,

intermediate and stiff matrix conditions (analysis of 10 approximately equally distributed segments per organoid for a total of 30 segments per datapoint). b hNTO radius growth rates at day 11 and corresponding matrix compressive stress states. Red curve represents best fit power curve ( $\mathrm{y}=51.4(-\mathrm{x})-0.291$ $\left.\mathrm{R}^{2}=0.2215\right)$ where knee point $\sigma_{\mathrm{rr}, \text { critical }}=-6.19 \mathrm{kPa}$, with fast growth rate regime $\sigma_{\mathrm{rr}}>\sigma_{\mathrm{rr} \text {, critical }}$ (open circles) and slow growth rate regime for $\sigma_{\mathrm{rr}}<\sigma_{\mathrm{rr}, \text { critical }}$ (black circles). c Compressive stress states for different stiffnesses at day 11 (data from a, statistical analysis was determined by unpaired two-sided t-test with Welch's correction ( $2 \mathrm{kPa} p=0.0006,8 \mathrm{kPa} p=0.0063)$ ). Error bars are SEM, scalebar $25 \mu \mathrm{m}$.

proliferation were upregulated upon stretch on both day 5 and day 11 (Supplementary Fig 13), confirming immunohistochemistry results (Supplementary Fig 7).

To systematically determine the transcriptional changes occurring upon stretch, differential gene expression analysis was performed followed by Gene Ontology (GO) enrichment analysis between conditions at day 11 (Fig. 4g). We identified extracellular matrix production, actin and cytoskeleton organization, as well as planar cell polarity (PCP) as processes upregulated in stretched samples, highlighting their role in stretch-mediated mechanotransduction and patterning regulation.

To explore transcriptional differences in a domain-specific manner, cells were binned according to their dorsoventral (D-V) geneset scores and their proportions were evaluated at days 5 and 11 (Fig. 4h). The ventral fraction in stretched organoids was reduced by 2.6 and 2.7 fold at days 5 and 11, respectively, suggesting that patterning was accompanied by a decrease in the number of FP domain cells, in line with morphological observations (Fig. 4h). To evaluate whether these results matched with in vivo development, we compared proportions of hNTO cells found in each $\mathrm{D}-\mathrm{V}$ domain with a published mouse neural tube scRNAseq dataset ${ }^{42}$. In day 11 hNTOs, stretch resulted in a reduction of the ventral domain, expansion of intermediate domain, and reduction of dorsal domain, similarly to trends observed during mouse neural tube development from E9.5 to E10.5 (Fig. 4i). Correlation analysis of hNTO fate proportions to that of the developing mouse neural tube revealed that unstretched hNTOs resembled the early E9.5 development stage, while stretched conditions were closer to the more developed E10.5/E11.5 neural tube. These results suggest that actuationmediated acceleration of patterning may stabilize more physiological domain proportions in hNTOs.

Because actuation changed domain-specific cell proportions, we next explored whether stretch-induced upregulation of processes related to ECM production, cytoskeleton remodeling, contractility and PCP exhibited domain-specific differential regulation (Fig. 4j). Contractility genes, such as ROCK1, MYLK, MYH9, DIAPH1, as well as ECM genes LAMA1, COL2A1, FN1, were upregulated before FP emergence, suggesting that these have a role in pattern induction. Conversely, RAC1, CDC42, PAK1, DIAPH1, involved in cytoskeleton organization were most expressed in the ventral domain at day 11 and were also the genes for which expression was most upregulated in this domain upon stretching, suggesting a role in pattern stabilization. Significant changes in PCP gene expression were also observed as a function of time, domain and mechanical state. Downstream PCP effectors DVL1 and SEC24B were most highly expressed immediately after stretch in the dorsal/intermediate domain whereas receptors VANGL1, CELSR1, and FZD1 were upregulated at day 11. Interestingly, DAAM1, which links PCP with the contractility machinery ${ }^{43}$ had highest expression in the ventral domain at day 11, but its expression also decreased the most in 
a

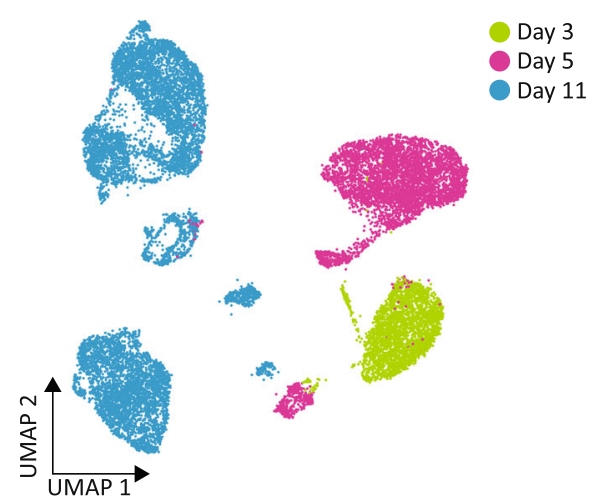

b

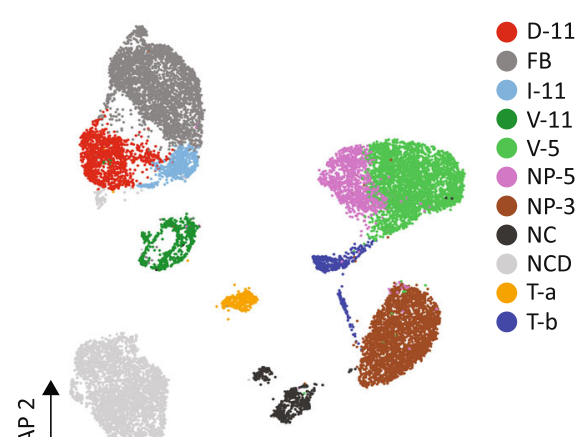

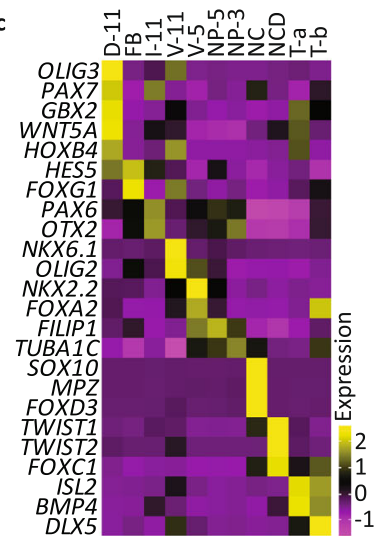

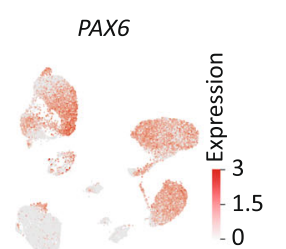

Ventral gene-set NKX6.1, OLIG2, NKX2.2 FOXA2

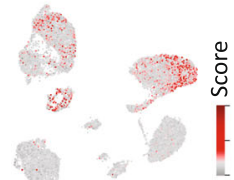

SOX1

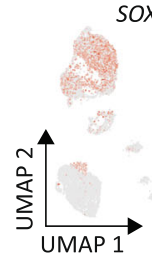

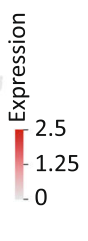

FOXA2 regulon
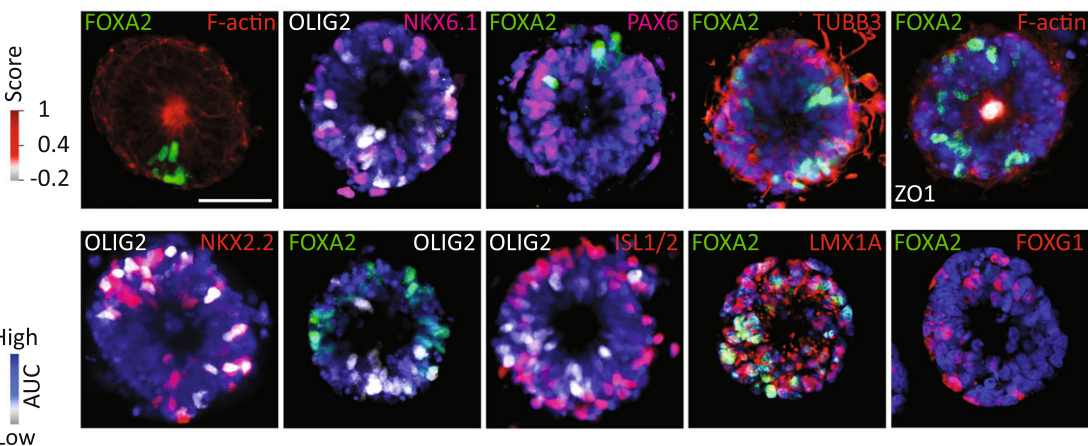

f

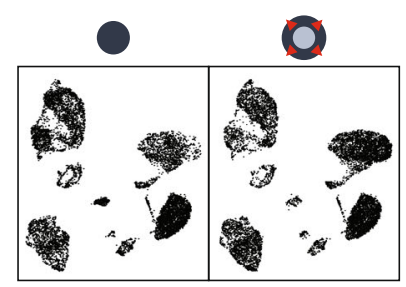

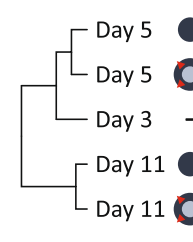

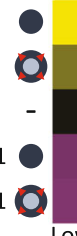

g

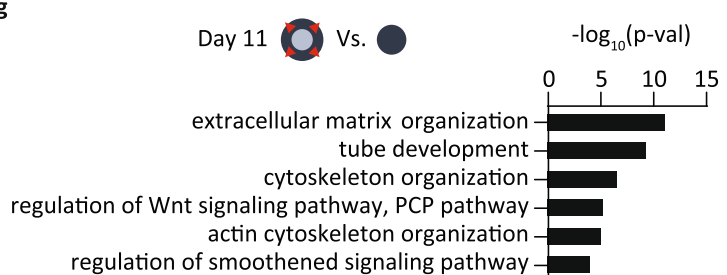

j

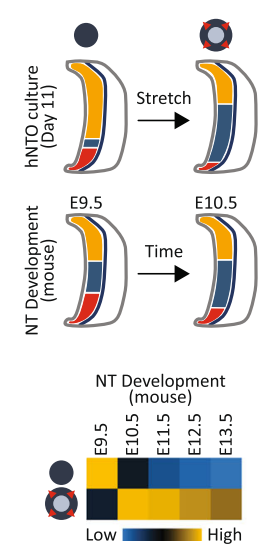

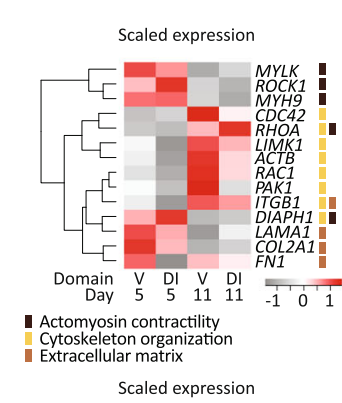

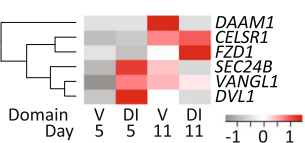

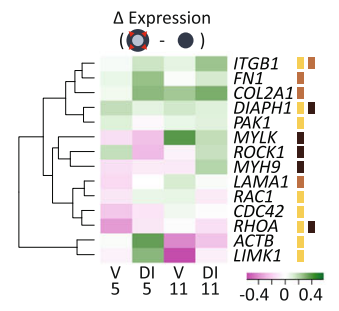

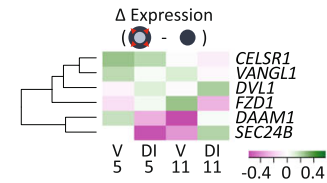

this domain upon stretch. Overall, these transcriptomic results suggest that pathways involved in contractility and PCP are likely mediators of patterning enhancement in actuated hNTOs.

Role of contractility and planar cell polarity in actuated hNTOs. In order to further investigate the role of contractility in FP induction and patterning we used the ROCK inhibitor Y-27632 (ROCKi) to perturb hNTO contractility and phosphorylated myosin light chain activity. Prolonged exposure to ROCKi reduced FP induction from 59 to $30 \%$ while patterning frequency of FOXA2 + hNTOs treated with ROCKi was insensitive to stretch (Fig. 5a). Lumen formation is a hallmark of apicobasal polarization in neuroepithelial cysts and is necessary for FP patterning in $\mathrm{mNTOs}^{25}$. We observed that the frequent perturbation of organoid lumens was a clear phenotypic effect of ROCKi on hNTOs. In contrast to the round lumen present in more than $90 \%$ of untreated organoids, only $55 \%$ of ROCKi- 
Fig. 4 Single-cell RNA-seq of hNTOs reveals actuation-specific transcriptomic changes. a UMAP of the combined dataset (day 3, with day 5 and day 11 stretched and unstretched samples). b UMAP of the combined dataset with identified clusters (Dorsal (D-11), forebrain (FB), intermediate (I-11), ventral (V-11), neuroprogenitors (NP-5 and NP-3), neural crest (NC), neural crest derivatives (NCD), and transition clusters (T-a and T-b). Top marker genes for each cluster and unannotated clusters in (Supplementary Data 1 and Supplementary Fig. 7a-b). c Exclusive expression in specific clusters of selected marker genes for anteroposterior, dorsoventral, neural crest, neural crest derivative identities. Expression values are normalized and centered. $\mathbf{d}$ UMAPs with $P A X 6$, early neural and intermediate dorsoventral marker, expression in multiple clusters, and SOX1, late neural progenitor marker, expression limited to day 11 clusters. UMAP showing highest ventral geneset scores in ventral clusters $\mathrm{V}-5$ and $\mathrm{V}-11$. Highest FOXA2 regulon activity in ventral and T-b clusters confirmed by AUC score. e Fate specification by IHC in unstretched hNTOs ( $n=3$, IHC for stretched hNTOs in Supplementary Fig. 10). f UMAPs for unstretched and stretched hNTOs, with correlation heatmap and hierarchical clustering. $\mathbf{g}$ GO enrichment analysis of upregulated key processes include ECM, actin and cytoskeleton organization and PCP upon stretch in day 11 dataset. $\mathbf{h}$ Dorsoventral cell fractions, based on binned cell identities, with lower ventral fractions in stretched hNTOs compared to unstretched counterparts. i Comparison to mouse neural tube development. Diagrams display approximate dorsal, intermediate and ventral population fractions in stretched and unstretched hNTO cultures at day 11 and in mouse NT E9.5 and E10.5 as previously reported 42 . For mouse NT development the dorsal, intermediate and ventral progenitor domains are color coded, respectively, as yellow (roof plate to dp3), blue (dp4 to p2), and red ( 1 to FP). The reduced ventral and dorsal population fractions at the expense of intermediate cells upon stretch in hNTOs are comparable to the transition from E9.5 to E10.5 in mouse neural tube development. Correlation matrix mapping hNTO dorsoventral identity to mouse neural tube development. $\mathbf{j}$ Gene expression comparison for unstretched samples for contractility, cytoskeleton organization, ECM and PCP markers, and their modulation upon stretch. Expression values are normalized and centered. Scalebars $50 \mu \mathrm{m}$.

treated organoids presented a lumen, with most of these having an elongated shape (Fig. 5b) likely due to increased cytoskeleton fluidity resulting from impaired contractility. The importance of lumen formation was underscored by the observation that less than $5 \%$ of organoids without a lumen were able to form a FP (Fig. 5c). Reduced FOXA2 induction as a result of ROCKi treatment can therefore be attributed to reduced lumenogenesis. The lack of patterning enhancement in stretched ROCKi-treated hNTOs was not linked to defects in lumen formation, as these were not significantly different between ROCKi-treated stretched and unstretched samples (Fig. 5b).

Cytoskeletal remodeling has been shown to increase under mechanical stimulation ${ }^{44}$, and we therefore hypothesized that it may be impaired upon ROCKi treatment. To test this, we evaluated the ratio of F-actin intensities within the hNTOs (intensity ratio, IR) between stretched and unstretched samples as a surrogate for remodeling activity. Stretched hNTOs had increased IR values (IR $>1$ ) compared to unstretched controls (Fig. 5d), while this phenomenon was completely abrogated upon ROCKi exposure (IR 1), outlining a mechanism of stretchinduced patterning enhancement involving contractilitymediated cytoskeleton remodeling. Similar trends to ROCKi exposure were observed upon myosin II inhibition using small molecule Blebbistatin $(10 \mu \mathrm{M}$, days 3-11) (Supplementary Fig. 14), with even less FP induction and lumen formation compared to ROCKi treatment. These results underscore the requirement for an intact actomyosin machinery in order to induce FP identity and to engage with exogenous forces, which enhances patterning in hNTOs.

The physical and molecular events occurring during neural tube morphogenesis must be coordinated with the concurrent convergent extension of the embryo. PCP signaling directly links these two processes, and had been identified as a differentially expressed pathway in our GO enrichment analysis. To investigate the links between PCP and FP patterning, we used a hPSC line with point mutations in PCP genes CELSR1, VANGL1, and $S E C 24 B^{45}$, which have also been linked to neural tube defects ${ }^{46-48}$. The use of a triple mutant line ensures that PCP is completely abrogated. Unlike control and ROCKi-treated hNTOs, $\mathrm{PCPKO}^{\mathrm{K}}$-hTOs exhibited the complete absence of FOXA2 (Fig. 5e) and high expression of PAX6 in both stretched and unstretched conditions (Supplementary Fig. 15). The complete lack of FP in $\mathrm{PCPKO}_{-h N T O s}$ indicates that PCP plays an early role in FP induction, rendering the cells unresponsive to the RASAG patterning signal. Similar to ROCKi-treated hNTOs, $\mathrm{PCPKO}^{\mathrm{K}} \mathrm{hNT}$ s exhibited distorted cytoskeletons, marked by lower LFs, high LARs and unchanged IR values upon stretch (Fig. 5f and Supplementary Fig. 16), suggesting that PCP impairment reduced organoid-wide response to exogenous mechanical modulations. The cytoskeleton disorganizations observed in both ROCKi/Blebbistatin-treated and PCPKO hNTOs and consequent lack of patterning enhancement in the former upon stretch suggest that the formation of organized epithelia is a critical requirement in hNTOs for responsiveness to patterning signals and their modulation by mechanical forces.

Altogether, our results demonstrate that organoids are responsive to exogenous mechanical modulation driven by matrix actuation. We show that human neural tube organoids have increased patterning events upon stretch through changes in their actomyosin contractility, cytoskeleton remodeling and PCP machineries (Fig. 6). We further show that this response depends on stretch magnitude and timing, as well as on an interplay with the extracellular matrix, whose stiffness must be tuned to optimally relay extrinsic forces. We identify parallels between the molecular behavior of actuated hNTOs and the morphological dynamics of in vivo neural tube development ${ }^{12}$. Our results suggest that mechanical forces in early human neural tube bending regulate the emergence of the floor plate and help orchestrate domain specification. The precise mechanisms leading to enhanced patterning upon actuation remain to be elucidated. It is possible that actuation confers developmental robustness and that fluctuations in contractility or PCP gene expression may be buffered by tissue-scale mechanical coupling, as has recently been shown during drosophila gastrulation 49,50 . The engineered platform we describe here to mechanically actuate $3 \mathrm{D}$ organoids, and the accompanying single-cell atlas, can be used in conjunction with synthetic matrices to completely specify the extrinsic mechanical state of organoids. Enlarging the toolbox of organoid biology to include actuation will allow for more robust and reliable $3 \mathrm{D}$ phenotypes, and will allow to interrogate the role of mechanotransduction in other developmental and disease model systems.

\section{Methods}

Human PSC lines. The human PSC lines used in this study were (1) ZO1 hPSC line (Mono-allelic mEGFP-Tagged TJP1 WTC hPSC Line, Coriell institute for Medical Research), (2) hPSC line reported in Sahakyan, V. et al. Sci Rep 8, 01821103 (2018), and (3) NCRM-1 (RRID:CVCL_1E71) hPSC line from NIH Center for Regenerative Medicine (CRM), Bethesda, USA.

Culture medium. Essential 8 (E8)-Flex Medium Kit (ThermoFisher Scientific) supplemented with $0.1 \%$ Penicillin Streptomycin (GIBCO) was used to maintain hPSC cultures. The neural differentiation medium comprised of 1:1 mixture of 

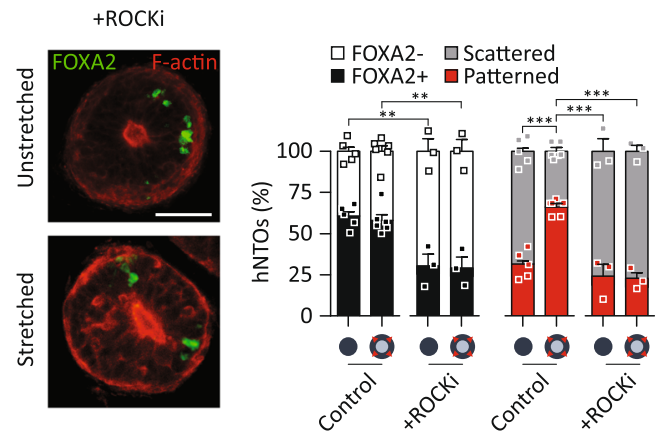

c
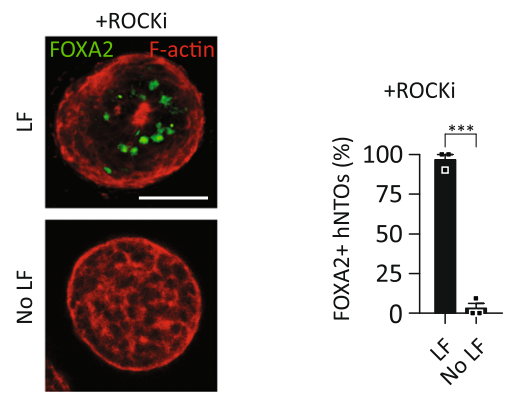
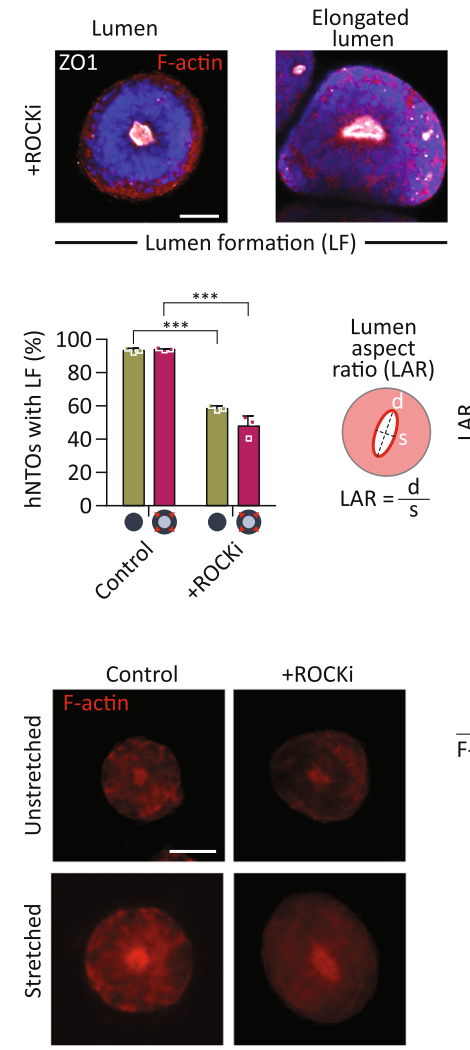

No lumen
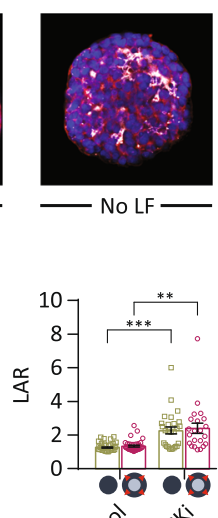

e
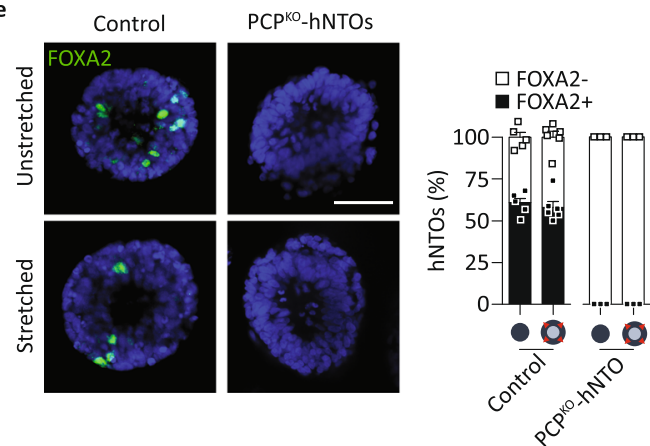
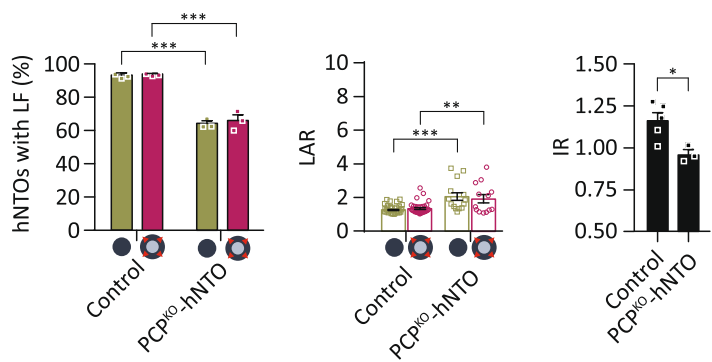

Fig. 5 Contractility and planar cell polarity determine floor plate induction and hNTO patterning. a Representative images of FOXA2 and F-actin expression in stretched and unstretched hNTOs with prolonged ROCKi exposure, and quantification of FP induction and patterning upon stretch compared to control conditions ( $n=3$ for unstretched (222) and stretched (187) hNTOs, control hNTOs from Fig. 1d, statistical analysis was determined by unpaired two-sided $t$-test (FOXA2+ control unstretched-ROCKi unstretched $p=0.0023, \mathrm{FOXA2}+$ control stretched-ROCKi unstretched $\mathrm{p}=0.0035$, patterned control stretched-control unstretched $\mathrm{p}<0.0001$, patterned control stretched-ROCKi unstretched $p=0.0006$, patterned control stretched-ROCKi

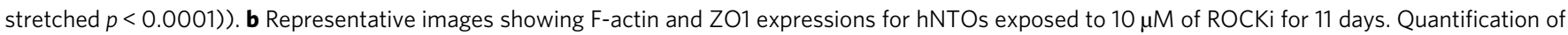
the number of hNTOs exhibiting lumen formation (LF) and lumen aspect ratio (LAR) ( $n=3$ for $>25$ hNTO per condition, statistical analysis was determined by unpaired two-sided $t$-test for LF (unstretched $p<0.0001$, stretched $p=0.0007$ ) and unpaired two-sided $t$-test with Welch's correction for LAR (unstretched $p<0.0001$, stretched $p=0.0018)$ ). c Representative images and quantification showing FOXA2 expression in hNTOs with and without LF ( $n=3$ for $>30$ hNTOs, statistical analysis was determined by unpaired two-sided $t$-test $p<0.0001$ ). d Representative hNTO images indicating F-actin expression, with quantification of F-actin intensity within the organoid. Intensity ratio (IR) quantifies the relative F-actin intensity within organoids between stretched and unstretched samples ( $n=3$ for $>25$ hNTOs per condition, statistical analysis was determined by unpaired two-sided $t$-test $p=0.0382$ ). e Representative images and quantification highlighting the absence of FOXA2 expression in PCPKO-hNTOs compared to control hNTOs under unstretched and stretched conditions ( $n=3$ for $>50$ PCPKO-hNTOs, control hNTOs from Fig. 1d). f Quantification of LF, LAR, and IR for PCPKO-hNTOs ( $n=3$ for $>30$ PCPKO-hNTOs per condition, statistical analysis was determined by unpaired two-sided $t$-test for LF (unstretched $p<0.0001$, stretched $p=$ 0.0008 ), by unpaired two-sided $t$-test with Welch's correction for LAR (unstretched $p=0.0037$, stretched $p=0.0011$ ), and by unpaired two-sided $t$-test for IR $p=0.0273)$ ). Error bars are SEM, scalebars $50 \mu \mathrm{m}$. 


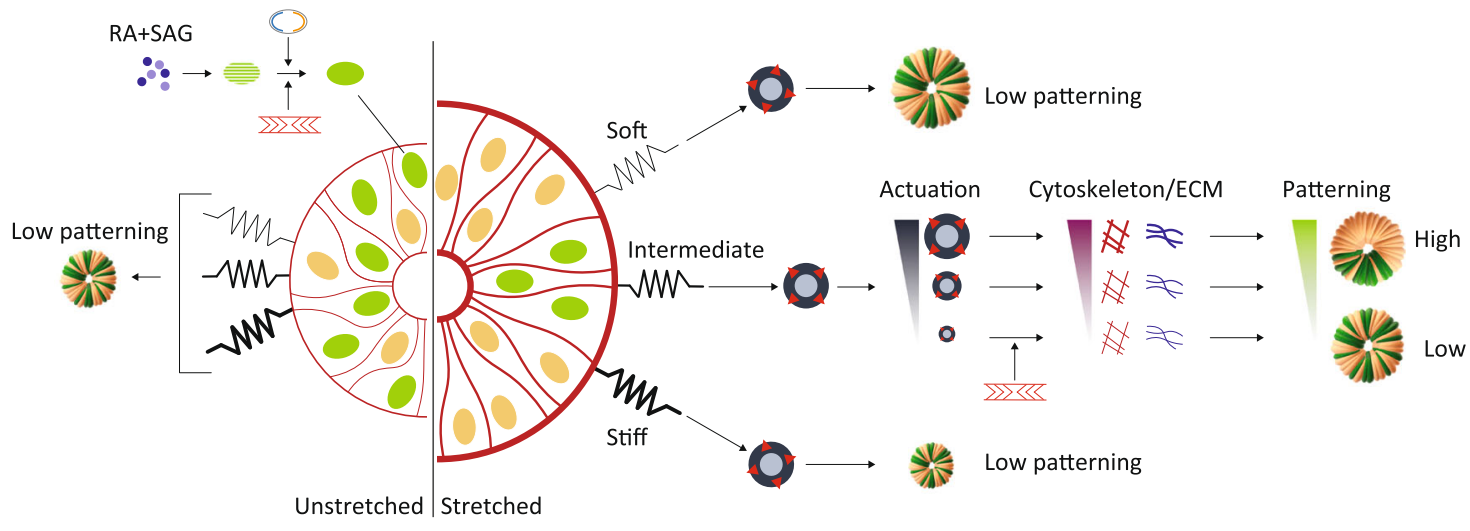

$\begin{array}{lll}\because \text { RA-SAG } & \text { FOXA2 } & \text { Contractility } \\ \text { OPCP } & \text { Matrix stiffness } & \text { Cytoskeleton } \\ \text { FOXA2 } & \text { O Actuation } & \text { ECM }\end{array}$

Fig. 6 Proposed model for floor plate induction and patterning in hNTOs. Induction of the FP requires active contractility and PCP. FP patterning enhancement relies on hNTO actuation in a matrix stiffness-dependent manner. Soft and stiff matrices result in no FP patterning enhancement upon actuation. In intermediate stiffness, actuated hNTOs exhibit higher FP patterning events linked to increased cytoskeleton and ECM remodeling activity.

neurobasal medium (GIBCO) and DMEM/F12 (GIBCO), 1\% N2 (GIBCO), 2\% B-27 (GIBCO), $1 \mathrm{mM}$ sodium pyruvate MEM (GIBCO), $1 \mathrm{mM}$ glutamax (GIBCO), $1 \mathrm{mM}$ nonessential amino acids (GIBCO), and $0.2 \%$ Penicillin Streptomycin (GIBCO).

Human PSC culture. Human PSCs were cultured in Matrigel-coated 6-well plates to $60-70 \%$ confluence. To passage the colonies, a 4 min treatment of Dispase (Sigma) was applied at $37^{\circ} \mathrm{C}$, followed by three PBS washes. $1 \mathrm{~mL}$ of E8-Flex medium supplemented with Y-27632 Rock inhibitor ROCKi (Hellobio) at $10 \mu \mathrm{M}$ was added and the colonies were scraped and gently agitated to break the colonies. The colonies were then split 1:6 and incubated in $2 \mathrm{~mL}$ of E8-Flex medium supplemented with ROCKi at $10 \mu \mathrm{M}$ for $24 \mathrm{~h}$. The medium was then replaced by $4 \mathrm{~mL}$ of ROCKi-free E8-Flex medium and incubation was resumed for $48 \mathrm{~h}$, at which point the colonies reached $60-70 \%$ confluence and were ready for passaging.

Human NTO culture in PEG hydrogels. At a confluence of $60-70 \%$, hPSCs were washed three times with PBS and dissociated into single cells by incubation in $1 \mathrm{~mL}$ of TrypLE Express (GIBCO) at $37^{\circ} \mathrm{C}$ for $3 \mathrm{~min}$. Subsequently, $9 \mathrm{~mL}$ of DMEM/F12 media supplemented with $20 \%$ FBS (GIBCO) was added and the cells were centrifuged at $300 \mathrm{RCF}$ for $3 \mathrm{~min}$. The supernatant was discarded and replaced by a second wash of $10 \mathrm{~mL}$ of DMEM/F12 media containing 20\% FBS. Cells were counted and remaining cells were centrifuged again at $300 \mathrm{RCF}$ for $3 \mathrm{~min}$. The pellet was resuspended in neural differentiation medium containing $10 \mu \mathrm{M}$ of ROCKi to obtain a cell density of $3.5 \mathrm{M}$ cells per $\mathrm{mL}$. The cells were added to a nondegradable (nd)PEG premix to constitute $10 \%$ of the hydrogel's total volume. Fifteen microliter droplets of cell-hydrogel mix were placed on the surface of elastomeric membranes that had previously undergone silanization through a nitric acid and (3-Aminopropyl) triethoxysilane (Sigma-Aldrich) treatment, and were mounted on equibiaxial or uniaxial stretching devices. Control membranes were placed inside a $10 \mathrm{~cm}$ culture dish. After gelation was visibly confirmed, an additional 20 min waiting time ensured complete gelation, and was followed by the addition of $2.5 \mathrm{~mL}$ of neural differentiation medium supplemented with $10 \mu \mathrm{M}$ of ROCK inhibitor. After $72 \mathrm{~h}$ the medium was replaced with $7 \mathrm{~mL}$ of neural differentiation medium supplemented with retinoic acid (RA) (Stemcell Technologies) at $0.25 \mu \mathrm{M}$ and smoothened agonist (SAG) (Stemcell Technologies) at $1 \mu \mathrm{M}$. The medium was fully refreshed every 2 days until endpoint at day 11. Increasing the medium volume from 2.5 to $7 \mathrm{~mL}$ ensured that media levels did not drop below the height of the PEG droplets upon stretch.

To change the RA-SAG treatment window along the experimental timeline, the treatment was applied for days $1-3$, days $3-5$, days $5-7$, days $7-9$, and days $9-11$ using a 96-well plate with $5 \mu \mathrm{L}$ hydrogel droplets and $200 \mu \mathrm{L}$ medium volume, following a 2 day media change protocol except for the earliest treatment. To apply prolonged ROCKi treatments, ROCKi was added to the medium at $10 \mu \mathrm{M}$ at day 0 and supplemented to all media changes until day 11. To inhibit myosin II, hNTOs were exposed to $10 \mu \mathrm{M}$ of Blebbistatin (Sigma-Aldrich) from day 3 (after the standard 3-day ROCKi treatment) until day 11.

PEG hydrogel preparation. PEG hydrogel precursors and buffers were prepared as previously described ${ }^{24,25}$. In brief, 8arm-PEG-vinylsulfone, $40 \mathrm{kDa}$ (PEG-VS) (NOF Corporation), was functionalized with FXIIIa-peptide substrates via Michael type addition. We have used a glutamine-containing peptide (NQEQVSPLERCG$\mathrm{NH} 2$ ) and lysine-containing peptides with a non-MMP-insensitive i.e., nondegradable sequence (AcFKGG-GDQGIAGF-ERCG-NH2). We obtained a glutamine-PEG precursor and a lysine-PEG precursor. Peptides were added to PEG-VS in a 1.2-fold molar excess over VS groups in $0.3 \mathrm{M}$ triethanolamine $(\mathrm{pH}$ 8.0 ) at $37^{\circ} \mathrm{C}$ for $2 \mathrm{~h}$, followed by dialysis (Snake Skin; MWCO 10k; Pierce) against ultrapure water for 4 days at $4^{\circ} \mathrm{C}$. After dialysis, the salt-free products were lyophilized to obtain a white powder. These functionalized PEG powders were then reconstituted in water to obtain a $10 \% \mathrm{wt} / \mathrm{vol}$ working stock solutions of PEG.

Factor XIII (FXIII) was reconstituted in water and activated with thrombin: 1 $\mathrm{mL}$ of FXIII (200 U/mL) and Fibrogammin P1250 (CSL Behring) were activated in the presence of $2.5 \mathrm{mM} \mathrm{CaCl} 2$ with $100 \mu \mathrm{L}$ of thrombin $(20 \mathrm{U} / \mathrm{mL}$; Sigma-Aldrich $)$ for $30 \mathrm{~min}$ at $37^{\circ} \mathrm{C}$. Aliquots of FXIIIa were stored at $-80^{\circ} \mathrm{C}$ for further use. Precursor solutions were prepared by stoichiometrically balanced $([\mathrm{Lys}] /[\mathrm{Gln}]=1)$ solutions of glutamine-PEG and lysine-PEG in Tris buffer (TBS; $50 \mathrm{mM}$; pH 7.6) containing $50 \mathrm{mM}$ calcium chloride. The crosslinking reaction was initiated by 10 $\mathrm{U} / \mathrm{mL}$ thrombin-activated FXIII and vigorous mixing.

The hydrogel composition (wt/vol of nd-PEG) to obtain various stiffnesses was the following: $0.7 \mathrm{kPa}: 1.25 \%, 2 \mathrm{kPa}: 1.5 \%, 8 \mathrm{kPa}: 3 \%$.

First, milliQ water, 10X Buffer and nd-PEG were mixed and kept at room temperature, until the hPSCs were prepared for embedding. After the addition of laminin (BD) to a final concentration of $0.1 \mathrm{mg} / \mathrm{ml}$ and $10 \mathrm{X}$ FXIII, the premix was ready for immediate cell embedding followed by plating. We have previously shown that laminin is covalently incorporated into the FXIII-crosslinked PEG and that the addition of $0.1 \mathrm{mg} / \mathrm{ml}$ laminin does not alter the mechanical properties of this matrix ${ }^{24}$

To supplement the hydrogels with red fluorescent beads (FluoSpheres Carboxylate-Modified Microspheres, $200 \mathrm{~nm}$, Invitrogen), the stock solution was first diluted 1:10 and then sonicated for $5 \mathrm{~min}$ to break up any aggregation prior to addition to the hydrogel premix for a final composition of $4 \%$ (1:250 dilution of the stock solution). Adding the fluorescent beads was done prior to the addition of the buffer solution step. The volume of fluorescent beads added was subtracted from the volume of milliQ water in the premix to ensure the total composition of the hydrogel was not compromised.

Stretching devices. An equibiaxial stretching device was used to apply equibiaxial strains on compatible elastomeric membranes hosting the hNTO containing hydrogels. Stretch was applied through the rotation of $8 \mathrm{arms}$ that connect to the membranes. The motor unit and the setup were placed in the incubator. The stretching schedule was controlled through an external computer. This allowed the modulation of stretch magnitude and duration.

A uniaxial stretching was also used to provide linear strains on the same membranes as the equibiaxial device. The apparatus connected to the membrane at two ends, one fixed and one free-moving. A motor unit translated the free-moving end thereby applying linear stretching. The entire instrument was placed in the incubator except for the programmable logic controller (ArduinoUno), which controls the rate and timing of stretching and remains outside the incubator.
Immunohistochemistry. Four percent paraformaldehyde (Sigma-Aldrich) was used to fix hNTOs for $2 \mathrm{~h}$ followed by washing with PBS. Permeabilization and blocking solutions were made with 0.3\% Triton X (PanREAC AppliChem) and $0.5 \%$ BSA solution (Sigma-Aldrich) for $30 \mathrm{~min}$. Primary antibodies (Supplementary Data 3) were suspended in permeabilization and blocking solution and applied 
to the fixed and permeabilized hNTOs for $24 \mathrm{~h}$. Three PBS washes were performed over an additional $24 \mathrm{~h}$ period. Subsequently, immunolabeling was performed over a duration of $24 \mathrm{~h}$ using donkey-anti mouse Alexa Fluor 555/647 (Invitrogen, dilution 1:500), donkey-anti rabbit Alexa Fluor 555/647 (Invitrogen, dilution 1:500), donkey-anti goat Alexa Fluor 555/647 (Invitrogen, dilution 1:500) and donkey-anti rat Alexa Fluor 647 (Jackson ImmunoResearch, dilution 1:500) secondary antibodies. To visualize filamentous actin we employed Alexa Fluor 647 conjugated phalloidin (Abcam, dilution 1:500). Hoechst was used to visualize nuclei. Click-it EDU Alexa Fluor 647 kit (Invitrogen) was used to label cells with active DNA synthesis. To visualize ZO1, the ZO1 reporter cell line was used.

Image acquisition and analysis. For quantification analysis, fluorescence images were obtained using an inverted microscope (Zeiss Axio Observer Z1; Carl Zeiss MicroImaging) equipped with a Colibri LED light source and a $\times 10$ air objective. Confocal representative images were obtained using a confocal microscope (Leica SP8 DIVE, Leica Microsystems) operated on either confocal or multiphoton mode with $\times 25$ water objective. Live imaging was performed using an inverted microscope (EVOS, Invitrogen) using a $\times 4$ and $\times 10$ air objectives under transmitted light or using an RFP filter for bead tracking experiments.

Possible differences in the stress states within the hydrogel along vertical axis are small as demonstrated by simulation (Supplementary Fig. 6c). However, to avoid any potential variations in stress, organoid settling within the hydrogel prior to polymerization restricted hNTO vertical positions to the bottom-most region, and therefore closest to membrane surface. This ensured that all analyzed hNTOs were subjected to comparable stress states when stretched and that the imposed strains most reflected that of the membrane.

Organoid size evaluation. To evaluate hNTO size, brightfield images were analyzed using ImageJ (Version 1.52a) by manual tracing of organoid borders to obtain an area, which was then converted to an equivalent diameter.

Patterning assessment in hNTOs. To evaluate FP domain size, fluorescence images were analysed using ImageJ by manually tracing regions that span FOXA2 + cells within hNTOs resulting in a FOXA2+ area. To obtain FOXA2 AR, FP domain size was divided by hNTO area. To systematically determine the scattering or patterning of FP domain within an hNTO, (1) the FOXA2 AR was determined, and (2) a FOXA2 intensity ratio (FIR) was obtained where the average FOXA2 intensity of the FOXA2+ region was first subtracted by the average FOXA2 intensity over the entire hNTO area, then divided by the latter. Subsequently, an hNTO was evaluate as scattered for 1) AR $>0.5$, and (2) AR $<0.5$ but FIR $<20 \%$. By contrast, an hNTO was evaluated as patterned for AR $<0.5$ and FIR $>20 \%$. These criteria were determined upon visual investigation of multiple FOXA2 expressions. To evaluate positional information of labeled samples, the line section in ImageJ was used. Although area quantification is used here, by using wide field microscopy we capture the majority of signal from the organoid volume allowing us to distinguish between patterned and scattered expressions of FOXA2.

Bead tracking experiments. Bead tracking experiment involved tracking fluorescent beads (FluoSpheres Carboxylate-Modified Microspheres, $200 \mathrm{~nm}$, Invitrogen) embedded within the matrix $(1: 250 \mathrm{v} / \mathrm{v})$. Imaging was performed every 2 days starting from day 1 . To assess matrix stretch and stress states in the vicinity of growing hNTOs, ImageJ was used where, at day 11, a segment normal to the border of the hNTO at its widest projected area, in the radial direction of stretching, and linking a bead pair in the same focal plane is first identified. Beads chosen close to day 1 organoids can often not be tracked in subsequent days since they are forced out of the focal plan by the growing organoids or become stuck to the surface of the hNTOs. Therefore, the bead pairs were chosen such that their final position is close $(<0.5 R$, where $R$ is the radius of the organoid) to the day 11 organoids to ensure stretch values are in the vicinity of the growing organoids. This also allowed easy tracking of the beads. Each bead pair is tracked over the 11 day protocol and for every other day, the new length, $L$, is divided by the reference length $L_{\mathrm{i}}$ (interbead length at day 1) to evaluate the stretch component in the direction of stretching $\lambda_{\mathrm{rr}}$ $=L / L_{\mathrm{i}}$, namely the interbead stretch. The matrix along the stretch direction is considered in tension for $\lambda_{\mathrm{rr}}>1$, in compression for $\lambda_{\mathrm{rr}}<1$, and neutral for $\lambda_{\mathrm{rr}}=1$. Given the expected large deformations with a stretch up to $\lambda_{\mathrm{rr}} \sim 1.40$, we employed a Neo-Hookean incompressible hyperelastic material model where $\lambda_{\mathrm{rr}}$ values were converted to the stress component in the direction of stretching using the following relation,

$$
\sigma_{\mathrm{rr}}=2 C_{10}\left(\lambda_{\mathrm{rr}}^{2}-\frac{1}{\lambda_{\mathrm{rr}}^{4}}\right)
$$

where $C_{10}=\mu / 2$, where $\mu$ is the shear modulus that relates to the elastic modulus, $E$, and Poisson's ratio, $v$, through $\mu=E /(2(1+v))$. We assumed the incompressibility of the hydrogels. and used a Poisson's ratio $v=0.5$. For each hNTO, 10 approximately equally distributed segments (bead pairs) around the organoid are observed and analyzed to provide an average stress state for the surrounding matrix. To determine the critical stress point $\sigma_{\text {rr critical }}$ (Fig. 3b), the MATLAB (R2018a, The MathWorks Inc.) Knee Point function (Version 1.1.0.0) was used for analysis.
PEG hydrogel equibiaxial simulations. Equibiaxial stretching of PEG hydrogels was simulated using the Finite Element Analysis (FEA) software Abaqus (Dassault Systemes, 2019). An axisymmetric model was created of the PEG matrix droplet attached to the membrane. The interface between the droplet and membrane was considered to be fully bonded (no relative motion). Symmetry conditions were imposed to the symmetry axis (zero radial displacements) and zero axial (vertical) displacements were imposed to the bottom surface of the membrane. To simulate stretching, a radial (horizontal) displacement was imposed at the right side of the membrane (side away from the symmetry axis). This displacement corresponded to a radial stretch of $\lambda_{\mathrm{rr}}=1.22$ and 1.41 as a result of increasing the membrane area by $50 \%$ and $100 \%$, respectively. To account for the expected large deformations, we employed the set of equations used by the Dynamic-Explicit Abaqus solver with nonlinear geometry (Nlgeom) to evaluate stresses in the droplets. In addition, we assumed material properties to be hyperelastic and employed a Neo-Hookean model. The materials were assumed as incompressible with Poisson's ratio $v=0.5$ and Neo-Hookean coefficient $D_{1}=0$. The Neo-Hookean coefficient $C_{10}$ for the different PEG hydrogels was calculated to be 116.7, 333.3, and 1,333.3 Pa for PEG elastic moduli $E=700 \mathrm{~Pa}, 2000 \mathrm{~Pa}$, and $8000 \mathrm{~Pa}$, respectively, using the relation $C_{10}=E /(4(1+v))$. With an elastic modulus much higher than the PEG hydrogels, $E=100,000 \mathrm{~Pa}$, the silicon rubber membrane $C_{10}$ was evaluated to be $16,666.7 \mathrm{~Pa}$. To compare simulation stress, $\sigma_{\mathrm{rr}, \text { simulation, }}$ with experimental stress, $\sigma_{\mathrm{rr}, \text { stress }}$, the bottom elements of the mesh ( 30 equally distributed mesh elements) in the simulated droplet were probed for $\sigma_{\text {rr simulation }}$ (stress in the direction of stretch), from the axis of symmetry to $75 \%$ of the radius of the droplet. This region maps to the same experimental zones where stress evaluation occurred for growing hNTOs and hNTO-free regions. The membrane part was hidden for visualization purposes (Supplementary Fig. 6).

Human NTO isolation and single-cell RNA sequencing. Organoids were grown following the hNTO differentiation protocol under five conditions (1) equibiaxially stretched-Day 11, (2) unstretch-Day 11, (3) equibiaxially stretched-Day 5, (4) unstretched-Day 5, and (5) hNTOs grown for 3 days (before stretching and RASAG treatment). For dissociation, the PEG gels were gently detached from the elastic membranes with a pipette tip and transferred to a clean and dry petri dish. A scalpel was used to slice the $15 \mathrm{uL}$ gel droplet into four to six pieces. A total of five $15 \mu \mathrm{L}$ gel droplets were collected, processed and pooled as described for each condition. Pooling was done to ensure enough cells were present for the library preparation and sequencing steps. Gel fragments were transferred to a $15 \mathrm{~mL}$ falcon tube containing $1 \mathrm{~mL}$ of prewarmed TrypLE Express at $37^{\circ} \mathrm{C}$. The samples were transferred to a warm water bath at $37^{\circ} \mathrm{C}$ for $7.5 \mathrm{~min}$ with gentle agitation every $1 \mathrm{~min}$ after the first $3 \mathrm{~min}$. Gradually, the gel fragments, as well as visible organoids, began to visually disappear. A visual inspection was performed using an inverted microscope to ensure the presence of single cells. The $1 \mathrm{~mL}$ solution was introduced to $9 \mathrm{~mL}$ of DMEM supplemented with 20\% FBS for TrypLE Express neutralization, and centrifuged at $500 \mathrm{RCF}$ for $5 \mathrm{~min}$. The pellet was resuspended in $200 \mathrm{uL}$ of N2B27 media and put on ice. This optimized dissociation protocol yielded an average viability of $85.4 \%$ across both stretched and control samples.

Library preparations for the scRNAseq was performed using 10X Genomics Chromium Single Cell 3' Kit, v3 (10X Genomics, Pleasanton, CA, USA). The cell count and the viability of the samples were assessed using LUNA dual fluorescence cell counter (Logos Biosystems) and a targeted cell recovery of 6000 cells was aimed for all the samples. Post cell count and QC, the samples were immediately loaded onto the Chromium Controller. Single-cell RNA-seq libraries were prepared using manufacturers recommendations (Single-cell 3' reagent kits v3 user guide; CG00052 Rev B), and at the different check points, the library quality was assessed using Qubit (ThermoFisher) and Bioanalyzer (Agilent). With a sequencing coverage targeted for 50,000 reads per cell, single-cell libraries were sequenced either on Illumina's NovaSeq 6000 or NovaSeq 500 platforms using paired-end sequencing workflow and with recommended 10X; v3 read parameters (28-8-0-91 cycles). The data generated from sequencing was demultiplexed and mapped against human genome reference using CellRanger v3.0.2.

Single-cell RNA sequencing data processing. We have sequenced 4694, 2541, 4687,6320 , and 5708 cells for day 3 , unstretched day 5 , stretched day 5 , unstretched day 11, and stretched day 11 samples, respectively, for a total of 23,950 cells, which were reduced after QC steps to 17,826 cells with an average of $4147 \pm 224$ detected genes per cell. Data manipulation and subsequent steps were performed using the Seurat ${ }^{51}$ tool for single-cell genomics version 3 in $\mathrm{R}$ version 3.4. A filtering step was performed to ensure the quality of the data, where the counts of mitochondrial reads and total genes reads were assessed. Cells with more than $15 \%$ of identifiable genes rising from the mitochondrial genome were filtered out. Similarly, cells having fewer identifiable genes than 200 (low quality) and above 7500 (probable doublets) were filtered out. Data normalization was performed, followed by the identification of 2000 highly variable genes using the FindVariableFeatures. S-phase and G2M-phase cell cycle regression was performed to allow cell clustering purely on cell identity and fate, which otherwise was biased by cell cycle phases. Auto scaling of the data was performed and described using principal component analysis (PCA) using the RunPCA function. 
Data clustering. Graph-based clustering using the FindNeighbors function (using top 15 principal components $(\mathrm{PCs})$ ) and FindCluster function (resolution $=0.5$ ) was performed to group cells based on their transcriptional profiles. No batch correction was performed on the dataset. Data visualization was performed using the Uniform Manifold Approximation and Projection (UMAP) dimensionality reduction technique using the RunUMAP package while employing the top 15 PCs identified in the previous PCA step. Cluster annotation was aimed at identifying D$\mathrm{V}$ regions based on the expression level of several hallmark genes related to anteroposterior (A-P) as well as dorsoventral (D-V) regionalization. The hallmark genes were grouped to create genesets for Forebrain (FB) (FOXG1, LHX2, DLX2, NKX2.1, GSX2, SIX3), Midbrain (MB) (PAX5, LMX1A, LMX1B, SIM1, EN1, EN2), Hindbrain (HB) (GBX2, HOXA2, HOX4, HOXB2, HOXB4), Dorsal (D) (LMX1A, OLIG3, PAX3, PAX7), Intermediate (I) (PAX6, DBX1, DBX2), and Ventral (V) (NKX6.1, OLIG2, NKX2.2, FOXA2). Using these genesets, we performed the following cluster identification: D-11 (dorsal with hindbrain, day 11), I-11 (intermediate with midbrain, day 11), FB (forebrain and mostly ventral, day 11), V-11 (ventral and mixed A-P, day 11), V-5 (ventral with forebrain and midbrain, day 5), NP-5 (neuroprogenitors, with forebrain and midbrain, day 5), and NP-3 (neuroprogenitors with forebrain and midbrain, day 3). These genesets were used to obtain a score for every cell using the AddModuleScore function. Other markers were included to identify additional clusters that did not exhibit the abovementioned genesets, such as Neural Crest (NC) markers (SOX10, MPZ, FOXD3), Neural Crest Derivative cells (NCD) (TWIST1, TWIST2, FOXC1). These markers were used to identify the NC (mixed days) cluster as well as broadly define the NCD (day 11) cluster which is a merger of three sperate subclusters. Clusters that did not have particular strong expression of the above markers were designated transition clusters (T-a, and T-b with mixed days) as they seemed to link the datasets of days 3 and 5 to those of day 11 . As such, these clusters were enriched in multiple markers, for example neuronal markers ISL2 as well as important dorsal signaling marker BMP4 in T-a and T-b, FOXA2 in T-b, and HOXB4 in T-a. One cluster was manually removed due to very low unique molecular identifier (UMI) count, after which the data were reprocessed to account for the removal of the cluster.

SCENIC. Transcription factor network inference was performed using the SCENIC $^{40}$ pipeline on the combined scRNAseq dataset. Regulon network activity was evaluated and represented by the scoring step AUCell for each cell using default settings on the full dataset. The AUC values for the FOXA2 regulon were implemented in the Seurat data matrix file and used for visualization using UMAPs.

Pseudotime trajectories. Trajectory analysis was performed using Monocle $2^{52}$ We performed the analysis on the BIOMEX platform (VIB-KU Leuven Center for Cancer Biology, BIOMEX) by importing the Seurat obtained normalized count matrix, metadata and using the 2000 Seurat obtained highly variable genes under default settings.

Correlation analysis. Correlation heatmap between stretch conditions was performed on the BIOMEX platform using the Seurat obtained normalized count matrix, metadata and using the 2000 Seurat obtained highly variable genes under default settings.

D-V cell binnning. To explore cell abundance along the $\mathrm{D}-\mathrm{V}$ axis, we assigned each cell an A-P and a D-V position using the combined dataset. Through this cell binning step, the A-P assignment was based the FB, MB, and $\mathrm{HB}$ genesets, while the $\mathrm{D}-\mathrm{V}$ one was based on the $\mathrm{D}, \mathrm{I}$ and $\mathrm{V}$ genesets. Using the AddModuleScore function, a cell was assigned the A-P position (FB, MB, or HB) that scored highest for the respective geneset. In a similar manner the $\mathrm{D}-\mathrm{V}$ position $(\mathrm{D}, \mathrm{I}$, and $\mathrm{V}$ ) was assigned. We only retained cells with positive assignment of A-P and D-V geneset scores and only those that could be assigned a position on both axes. Accounting for the A-P score ensured the D-V assignment was biologically relevant. Finally, to obtain the $\mathrm{D}-\mathrm{V}$ fractions we divided the amount of cells belonging to each assigned position $(\mathrm{D}, \mathrm{I}$, or $\mathrm{V})$ by the total cells along the $\mathrm{D}-\mathrm{V}$ axis. The process was conducted for samples of days 5 and 11 for both stretch conditions.

\section{Differential gene expression analysis (DGEA), gene ontology (GO) enrich-} ment analysis, and proliferation geneset. To perform a DGEA we employed the FindMarkers function, a part of the Seurat R package, between all day 11 cells, with function parameters min.pct $=0.0$ and logfc.threshold $=0.0$ to capture the complete gene list across the full range of LFC.

To perform the GO enrichment analysis, we first extracted the full list of differentially expressed genes ranked by LFC from highest to lowest. This list was then inserted into the GOrilla ${ }^{53}$ web portal using the Homo sapiens organism, single ranked file running mode, and a $p$-value threshold of $10^{-4}$. The results were then passed to the REVIGO ${ }^{54}$ web portal to obtain a summarized list of gene ontology terms using the default setting and small similarity option. The gene ontology terms were then ranked by significance of which relevant processes were reported.

A proliferation geneset was created using known proliferation markers (MKI67, TOP2A, PCNA, BIRC5, CENPF, USP1, ATAD2, CDCA8, CDC42) and cell scores were obtained using the AddModuleScore function. The average scaled geneset expression per condition (day 3, day 5 unstretched, day 5 stretched, day 11 unstretched, day 11 stretched) was then generated.

Quantification and statistical analysis. For statistical analysis we employed a two-way ANOVA statistical test and an unpaired two-tailed $t$-test with correction where appropriate with a 95\% confidence interval (GraphPad Prism 6, Version 6.01, GraphPad Software, Inc.). When determining patterning and scattering significance between conditions, the statistical analysis was performed using the patterned values of the various conditions. Similarly when determining the FOXA2 \pm hNTOs, the statistical analysis was performed using the FOXA2+ hNTO values of the various conditions. Pearson correlations were performed to evaluate linear regression where appropriate. Statistical significance was considered for all comparisons with $p<0.05$. For hierarchical clustering, we employed the $\mathrm{R}$ package heatmap.2.

Reporting summary. Further information on research design is available in the Nature Research Reporting Summary linked to this article.

\section{Data availability}

All raw sequencing data, and the combined processed and metadata files generated in this study are available at GEO. The accession number for the reported data is (GSE154120). This study did not generate any unique code.

Received: 22 September 2020; Accepted: 3 April 2021; Published online: 27 May 2021

\section{References}

1. Heisenberg, C. P. \& Bellaïche, Y. Forces in tissue morphogenesis and patterning. Cell 153, 948-962 (2013).

2. LeGoff, L. \& Lecuit, T. Mechanical forces and growth in animal tissues. Cold Spring Harb Perspect. Biol. 8, a019232 (2015).

3. Miller, C. J. \& Davidson, L. A. The interplay between cell signalling and mechanics in developmental processes. Nat. Rev. Genet. 14, 733-744 (2013).

4. Loganathan, R. et al. Extracellular matrix motion and early morphogenesis. Development 143, 2056-2065 (2016).

5. Benazeraf, B. et al. A random cell motility gradient downstream of FGF controls elongation of an amniote embryo. Nature 466, 248-252 (2010).

6. Eiraku, M. et al. Self-organizing optic-cup morphogenesis in threedimensional culture. Nature 472, 51-56 (2011).

7. Okuda, S. et al. Strain-triggered mechanical feedback in self-organizing opticcup morphogenesis. Sci. Adv. 4, eaau1354 (2018).

8. Sato, T. \& Clevers, H. Growing self-organizing mini-guts from a single intestinal stem cell: mechanism and applications. Science 340, 1190-1194 (2013).

9. Shyer, A. E., Huycke, T. R., Lee, C., Mahadevan, L. \& Tabin, C. J. Bending gradients: how the intestinal stem cell gets its home. Cell 161, 569-580 (2015)

10. Colas, J. F. \& Schoenwolf, G. C. Towards a cellular and molecular understanding of neurulation. Dev. Dyn. 221, 117-145 (2001).

11. Vijayraghavan, D. S. \& Davidson, L. A. Mechanics of neurulation: from classical to current perspectives on the physical mechanics that shape, fold, and form the neural tube. Birth Defects Res. 109, 153-168 (2017).

12. Nikolopoulou, E., Galea, G. L., Rolo, A., Greene, N. D. \& Copp, A. J. Neural tube closure: cellular, molecular and biomechanical mechanisms. Development 144, 552-566 (2017).

13. Kuzmicz-Kowalska, K. \& Kicheva, A. Regulation of size and scale in vertebrate spinal cord development. Wiley Interdiscip. Rev. Dev. Biol. 11, 383 (2020).

14. Turner, D. A. et al. Anteroposterior polarity and elongation in the absence of extraembryonic tissues and spatially localised signalling in Gastruloids, mammalian embryonic organoids. Development 26, 150391 (2017).

15. Grigoryan, B. et al. Multivascular networks and functional intravascular topologies within biocompatible hydrogels. Science 364, 458-464 (2019).

16. Bertassoni, L. E. et al. Hydrogel bioprinted microchannel networks for vascularization of tissue engineering constructs. Lab Chip 14, 2202-2211 (2014).

17. $\mathrm{Xu}, \mathrm{C}$. et al. Freeform vertical and horizontal fabrication of alginate-based vascular-like tubular constructs using inkjetting. J. Manuf. Sci. Eng. https:// doi.org/10.1115/1.4028578 (2014).

18. Moroni, L. et al. Biofabrication strategies for $3 \mathrm{D}$ in vitro models and regenerative medicine. Nat. Rev. Mater. 3, 21-37 (2018)

19. Kratochvil, M. J. et al. Engineered materials for organoid systems. Nat. Rev. Mater. 4, 606-622 (2019).

20. Ehrbar, M. et al. Biomolecular hydrogels formed and degraded via site-specific enzymatic reactions. Biomacromolecules 8, 3000-3007 (2007). 
21. Gjorevski, N. et al. Designer matrices for intestinal stem cell and organoid culture. Nature 539, 560-564 (2016).

22. Brown, T. E. et al. Secondary photocrosslinking of click hydrogels to probe myoblast mechanotransduction in three dimensions. J. Am. Chem. Soc. 140, 11585-11588 (2018).

23. Cruz-Acuna, R. et al. Synthetic hydrogels for human intestinal organoid generation and colonic wound repair. Nat. Cell Biol. 19, 1326-1335 (2017)

24. Ranga, A. et al. 3D niche microarrays for systems-level analyses of cell fate. Nat. Commun. 5, 4324 (2014).

25. Ranga, A. et al. Neural tube morphogenesis in synthetic $3 \mathrm{D}$ microenvironments. Proc. Natl Acad. Sci. USA 113, E6831-E6839 (2016)

26. Hughes, A. J. et al. Engineered tissue folding by mechanical compaction of the mesenchyme. Dev. Cell 44, 165-178 (2018).

27. Demers, C. J. et al. Development-on-chip: in vitro neural tube patterning with a microfluidic device. Development 143, 1884-1892 (2016).

28. Rifes, P. et al. Modeling neural tube development by differentiation of human embryonic stem cells in a microfluidic WNT gradient. Nat. Biotechnol. 25, 020-0525 (2020)

29. Xue, X. et al. Mechanics-guided embryonic patterning of neuroectoderm tissue from human pluripotent stem cells. Nat. Mater. 17, 633-641 (2018).

30. Poling, H. M. et al. Mechanically induced development and maturation of human intestinal organoids in vivo. Nat. Biomed. Eng. 2, 429-442 (2018).

31. Sasai, N., Kutejova, E. \& Briscoe, J. Integration of signals along orthogonal axes of the vertebrate neural tube controls progenitor competence and increases cell diversity. PLoS Biol. 12, e1001907 (2014).

32. Zheng, Y. et al. Dorsal-ventral patterned neural cyst from human pluripotent stem cells in a neurogenic niche. Sci Adv. 5, eaax5933 (2019).

33. Ebisuya, M. \& Briscoe, J. What does time mean in development? Development 145, 164368 (2018)

34. Ybot-Gonzalez, P., Cogram, P., Gerrelli, D. \& Copp, A. J. Sonic hedgehog and the molecular regulation of mouse neural tube closure. Development 129, 2507-2517 (2002).

35. Zagorski, M. et al. Decoding of position in the developing neural tube from antiparallel morphogen gradients. Science 356, 1379-1383 (2017).

36. Uygur, A. et al. Scaling pattern to variations in size during development of the vertebrate neural tube. Dev. Cell 37, 127-135 (2016).

37. Yang, C., Tibbitt, M. W., Basta, L. \& Anseth, K. S. Mechanical memory and dosing influence stem cell fate. Nat. Mater. 13, 645-652 (2014).

38. Mathur, J., Shenoy, V. B. \& Pathak, A. Mechanical memory in cells emerges from mechanotransduction with transcriptional feedback and epigenetic plasticity. bioRxiv https://doi.org/10.1101/2020.03.20.000802 (2020).

39. Zhang, X. et al. Pax6 is a human neuroectoderm cell fate determinant. Cell Stem Cell 7, 90-100 (2010).

40. Aibar, S. et al. SCENIC: single-cell regulatory network inference and clustering. Nat. Methods 14, 1083-1086 (2017).

41. Dessaud, E., McMahon, A. P. \& Briscoe, J. Pattern formation in the vertebrate neural tube: a sonic hedgehog morphogen-regulated transcriptional network. Development 135, 2489-2503 (2008).

42. Delile, J. et al. Single cell transcriptomics reveals spatial and temporal dynamics of gene expression in the developing mouse spinal cord. Development 146, 173807 (2019).

43. Nishimura, T., Honda, H. \& Takeichi, M. Planar cell polarity links axes of spatial dynamics in neural-tube closure. Cell 149, 1084-1097 (2012).

44. Hoffman, L. M., Jensen, C. C., Chaturvedi, A., Yoshigi, M. \& Beckerle, M. C. Stretch-induced actin remodeling requires targeting of zyxin to stress fibers and recruitment of actin regulators. Mol. Biol. Cell 23, 1846-1859 (2012).

45. Sahakyan, V. et al. Folic acid exposure rescues spina bifida aperta phenotypes in human induced pluripotent stem cell model. Sci. Rep. 8, 018-21103 (2018).

46. Lei, Y. et al. Identification of novel CELSR1 mutations in spina bifida. PLoS ONE 9, e92207 (2014).

47. Greene, N. D., Gerrelli, D., Van Straaten, H. W. \& Copp, A. J. Abnormalities of floor plate, notochord and somite differentiation in the loop-tail (Lp) mouse: a model of severe neural tube defects. Mech. Dev. 73, 59-72 (1998)

48. Merte, J. et al. Sec24b selectively sorts Vangl2 to regulate planar cell polarity during neural tube closure. Nat. Cell Biol. 12, 41-46 (2010).

49. Eritano, A. S. et al. Tissue-scale mechanical coupling reduces morphogenetic noise to ensure precision during epithelial folding. Dev. Cell 53, 212-228 (2020).

50. Martin, E. \& Suzanne, M. When the community silences disruptive elements: multiscale mechanical coupling buffers morphogenetic imprecisions. Dev. Cell 53, 135-137 (2020).
51. Satija, R., Farrell, J. A., Gennert, D., Schier, A. F. \& Regev, A. Spatial reconstruction of single-cell gene expression data. Nat. Biotechnol. 33, 495-502 (2015).

52. Trapnell, C. et al. The dynamics and regulators of cell fate decisions are revealed by pseudotemporal ordering of single cells. Nat. Biotechnol. 32, 381-386 (2014).

53. Eden, E., Navon, R., Steinfeld, I., Lipson, D. \& Yakhini, Z. GOrilla: a tool for discovery and visualization of enriched GO terms in ranked gene lists. BMC Bioinformatics 10, 1471-2105 (2009).

54. Supek, F., Bošnjak, M., Škunca, N. \& Šmuc, T. REVIGO summarizes and visualizes long lists of gene ontology terms. PLoS ONE 6, 18 (2011).

\section{Acknowledgements}

This work was supported by the FWO grants G087018N and G0H6316N, FWO postdoctoral fellowships $1217220 \mathrm{~N}$ (A.A.) and 12ZR120N (M.C.), Interreg Biomat-on-Chip grant and Vlaams-Brabant and Flemish Government co-financing, KU Leuven grants C14/17/111 and C32/17/027, King Baudouin Foundation grant J1810950-207421, and Hercules infrastructure grant I009718N. R.F. and Y.L. were supported by NIH R01 grants HD095520, HD081216 and HD100535. M.B. was supported by the Postgraduate in Europe program funded by Fundación La Caixa LCF/BQ/EU17/11590066. We thank Gert Hulselmans, Jasmien Willems, and Vincent Merens for technical assistance.

\section{Author contributions}

A.A. conducted experiments and analysis. B.D., G.R., M.B., and S.P. conducted experiments. A.A., B.G., and D.R. developed the mechanical stretching instruments and associated protocols. Y.L., X.C., and R.F. characterized the PCPKO-hPSC line. A.A., S.P., K.D., and S.A. generated and analyzed the scRNAseq data. A.A., J.B., M.C., H.O., and A.R. analyzed and interpreted the bead tracking and FEA simulations. A.A., R.F., M.S., C.V., H.O., P.D., S.A., and A.R. interpreted the data and edited the manuscript. A.A. and A.R. wrote the manuscript.

\section{Competing interests}

R.F. was CEO of Teratomic Consulting, LLC, which has been dissolved. He is also an associate editor of the journal Reproductive and Developmental Medicine, which provides his travel expenses. The other authors declare no competing interests.

\section{Additional information}

Supplementary information The online version contains supplementary material available at https://doi.org/10.1038/s41467-021-22952-0.

Correspondence and requests for materials should be addressed to A.R.

Peer review information Nature Communications thanks the anonymous reviewer(s) for their contribution to the peer review of this work.

Reprints and permission information is available at http://www.nature.com/reprints

Publisher's note Springer Nature remains neutral with regard to jurisdictional claims in published maps and institutional affiliations.

Open Access This article is licensed under a Creative Commons Attribution 4.0 International License, which permits use, sharing, adaptation, distribution and reproduction in any medium or format, as long as you give appropriate credit to the original author(s) and the source, provide a link to the Creative Commons license, and indicate if changes were made. The images or other third party material in this article are included in the article's Creative Commons license, unles indicated otherwise in a credit line to the material. If material is not included in the article's Creative Commons license and your intended use is not permitted by statutory regulation or exceeds the permitted use, you will need to obtain permission directly from the copyright holder. To view a copy of this license, visit http://creativecommons.org/ licenses/by/4.0/.

(C) The Author(s) 2021 